\title{
Selective Ring Expansion-Alkylation of Formyl[2.2.1]bicyclic Carbinols with C-Nucleophiles: A Unique Route to Cyclopentane Derivatives
}

\author{
Te-Fang Yang*, Chih-Hao Tseng, Kuan-I Wu and Chung-Nien Chang \\ Department of Applied Chemistry, Chaoyang University of Technology, \\ Wufong, 413, Taichung, Taiwan, ROC
}

Table of contents

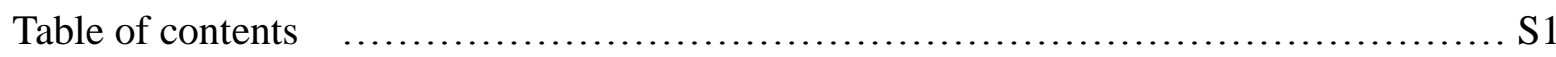

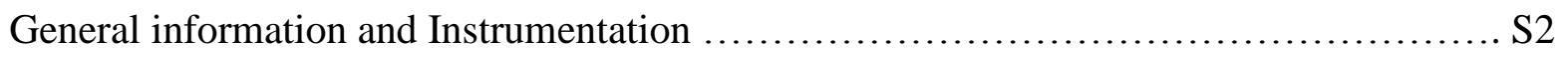

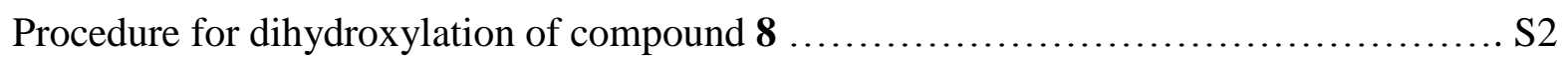

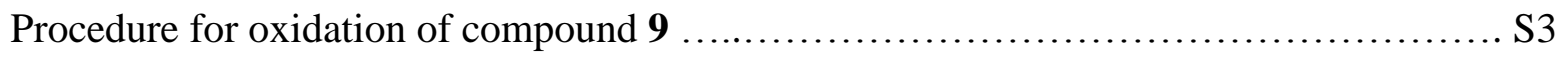

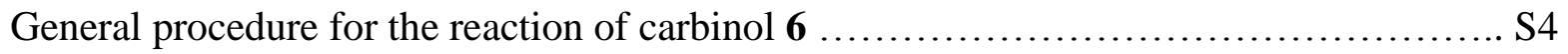

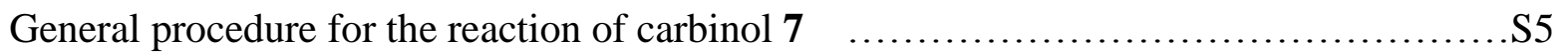

General procedure for the ring-cleavage of diols $\mathbf{1 0}, \mathbf{1 4 , 1 6}, \mathbf{1 7}$ and $21 . \ldots \ldots \ldots \ldots \ldots$......... 5

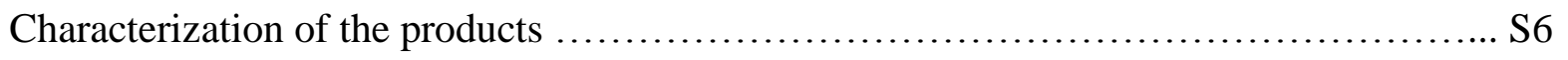

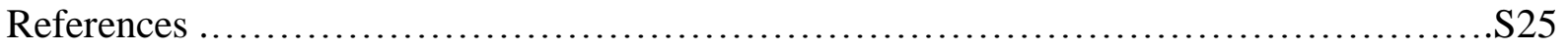


General information. Moisture-sensitive solvents were dried with standard methods and transferred via a syringe when necessary. Reactions were carried out in round bottom flasks fitted with rubber septa under argon. Crude product solutions were dried on $\mathrm{Na}_{2} \mathrm{SO}_{4}$ and concentrated with a rotary evaporator below $40{ }^{\circ} \mathrm{C}$ at $\sim 30$ Torr. Silica gel column chromatography was performed employing 230-400 mesh silica gel. TLC was performed on silica gel sheets with organic binder and detected by $0.5 \%$ phosphomolybdic acid solution in $95 \%$ ethanol. $\mathrm{MeMgBr}\left(3.0 \mathrm{M}\right.$ in $\left.\mathrm{Et}_{2} \mathrm{O}\right), \mathrm{EtMgBr}\left(1.0 \mathrm{M}\right.$ in THF), $\mathrm{CH}_{2} \mathrm{CHMgBr}\left(1.0 \mathrm{M}\right.$ inTHF), $\mathrm{CH}_{3} \mathrm{CCMgBr}$ (0.5 M in THF), $\mathrm{PhMgBr}\left(2.0 \mathrm{M}\right.$ in THF), cyclopentylMgBr (2.0 $\mathrm{M}$ in $\left.\mathrm{Et}_{2} \mathrm{O}\right), \mathrm{BnMgCl}(2.0 \mathrm{M}$ in $\mathrm{THF}), \mathrm{C}_{6} \mathrm{H}_{5} \mathrm{CCMgBr}$ (1.0 M in THF), AllylMgCl (2.0 M in THF), $i$-propylMgCl (2.0 $\mathrm{M}$ in Et $\mathrm{Et}_{2} \mathrm{O}$ ), $n$ - BuMgCl (2.0 M in THF), MeLi (1.6 M in $\left.\mathrm{Et}_{2} \mathrm{O}\right), n$-BuLi (1.6 M in hexane), PhLi (1.9 M in cyclohexane-ether), and tert-BuOK were purchased and used without further purification.

Instrumentation. An FT/IR spectrophotometer was used for obtaining the infrared spectra. Data are expressed as wave number of absorption $\left(\mathrm{cm}^{-1}\right) .{ }^{1} \mathrm{H} N M R$ and ${ }^{13} \mathrm{C}$ NMR spectra were obtained using a $200 \mathrm{MHz}$ spectrometer. Chemical shifts ( $\delta$ scale) are expressed in parts per million downfield from tetramethylsilane $(\delta=0.00) . \quad{ }^{1} \mathrm{H}$ NMR data are presented as follows: chemical shift, multiplicity ( $\mathrm{s}=$ singlet, $\mathrm{br} \mathrm{s}=$ broad singlet, $\mathrm{d}=$ doublet, $\mathrm{dd}=\mathrm{doublet}$ of doublet, $\mathrm{t}=$ triplet, $\mathrm{m}=$ multiplet and/or multiple resonances), coupling constant in $\mathrm{Hz}$ (Hertz), integration. Melting points were recorded with a Fargo (MP-1D) apparatus, and are uncorrected.

\section{Procedure for dihydroxylation of 8}

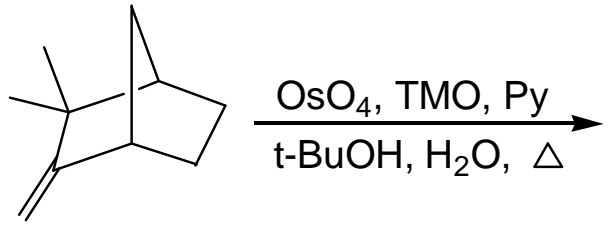

8

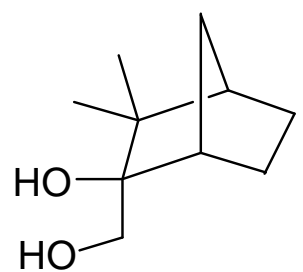

9

To a mixture of $\mathbf{8}(2.0 \mathrm{~g}, 14.7 \mathrm{mmol}), N, N$-dimethylmethanamine oxide (2.48 g, $22.3 \mathrm{mmol})$ in $t$ - $\mathrm{BuOH}(30 \mathrm{~mL})$, water $(5 \mathrm{~mL})$ and pyridine $(2.0 \mathrm{~mL})$ was added dropwise osmium tetroxide $(2.5 \%$ in $t-\mathrm{BuOH}, 1.48 \mathrm{~mL}, 0.114 \mathrm{mmol})$. The reaction mixture was heated at reflux for $5 \mathrm{~h}$, then cooled to room temperature, and quenched with sodium bicarbonate (20 $\%, 10 \mathrm{~mL}$ ). The mixture was extracted with petroleum ether, washed with water, dried $\left(\mathrm{Na}_{2} \mathrm{SO}_{4}\right)$, and concentrated under reduced pressure. The residue was purified with flash column chromatography $(1: 1$, hexane/EtOAc) to give $9(2.2 \mathrm{~g}, 90 \%$ yield, $90 \% \mathrm{de})$ as a white solid: $\mathrm{R}_{\mathrm{f}}=0.17\left(3: 1\right.$, hexane/EtOAc); $\mathrm{mp} 194-196^{\circ} \mathrm{C} ;[\alpha]_{\mathrm{D}}{ }^{27}=+12.1\left(0.01, \mathrm{CH}_{2} \mathrm{Cl}_{2}\right)$; IR (KBr) 3369 (br), 2963, 2881, $1464 \mathrm{~cm}^{-1} ;{ }^{1} \mathrm{H}$ NMR (200 MHz, $\mathrm{CDCl}_{3}$ ): $\delta 3.71$ (dd, J=6.0 $\mathrm{Hz}, 11.2 \mathrm{~Hz}, 1 \mathrm{H}), 3.58$ (dd, J=4.4 Hz, 11.2 Hz, 1H), 2.81(dd, J=4.4 Hz, $6.0 \mathrm{~Hz}, \mathrm{OH}), 2.48$ (s. $\mathrm{OH}), 2.13-1.11(\mathrm{~m}, 8 \mathrm{H}), 1.02(\mathrm{~s}, 3 \mathrm{H}), 0.93(\mathrm{~s}, 3 \mathrm{H}) ;{ }^{13} \mathrm{C} \mathrm{NMR}\left(50 \mathrm{MHz}, \mathrm{CDCl}_{3}\right): \delta 81.9$, 63.9, 49.7, 47.2, 43.4, 34.5, 25.3, 23.7, 22.7, 21.6. Anal. Calcd for $\mathrm{C}_{10} \mathrm{H}_{18} \mathrm{O}_{2}$ : C, 70.55; $\mathrm{H}$, 10.66. Found: C, 70.88; H, 10.36 . 

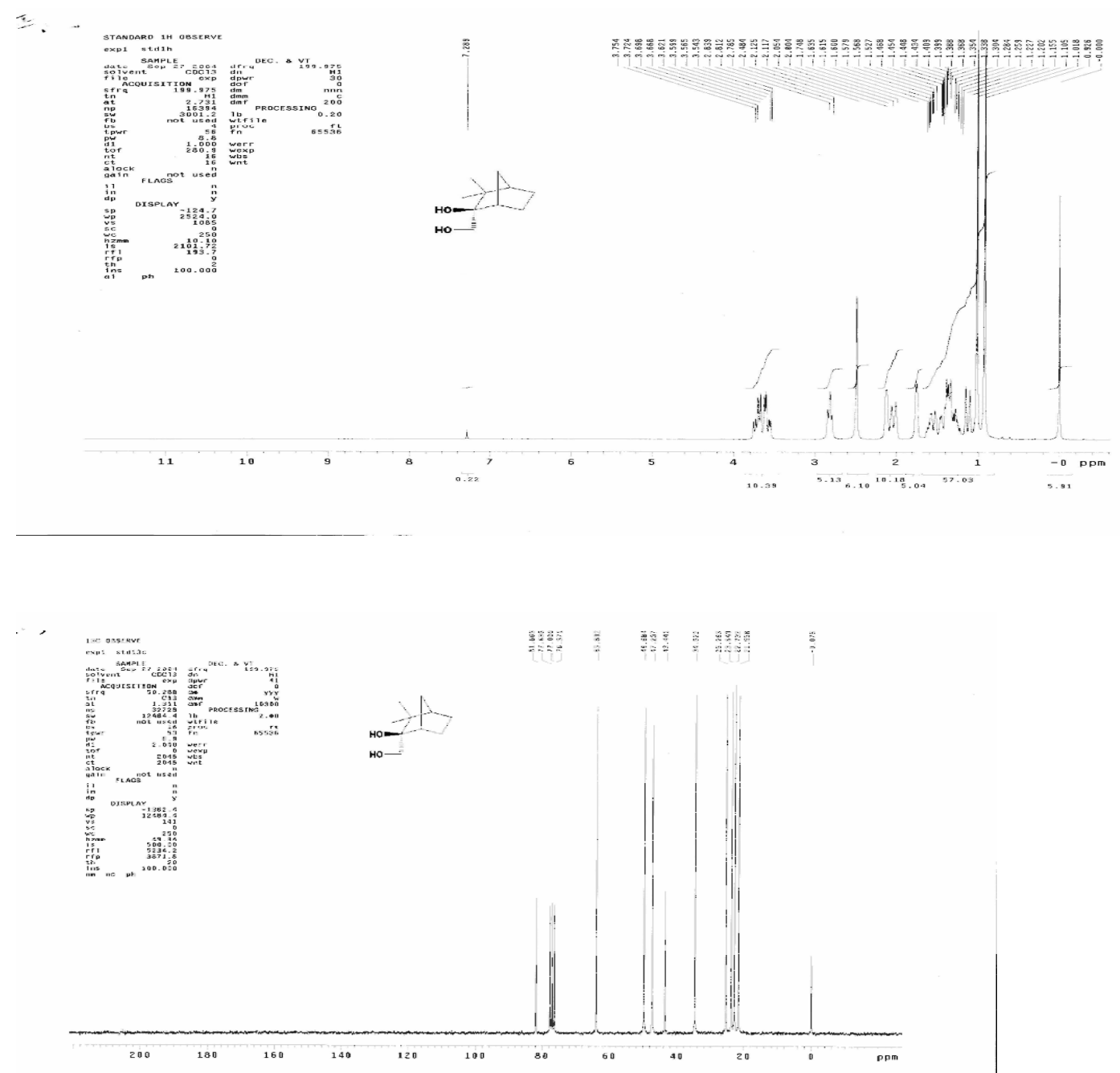

Procedure for preparation of 7.

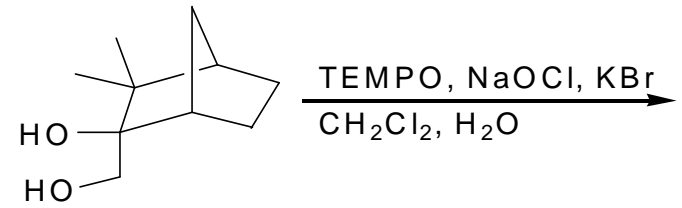

9

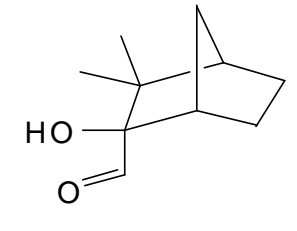

7

To a mixture of 9 (1.0 g, $5.87 \mathrm{mmol})$, TEMPO (0.03 g, $0.19 \mathrm{mmol}), \mathrm{KBr}(0.13 \mathrm{~g}, 1.10 \mathrm{mmol})$, $\mathrm{NaHCO}_{3}(0.13 \mathrm{~g}, 1.55 \mathrm{mmol})$ in $\mathrm{CH}_{2} \mathrm{Cl}_{2}(40 \mathrm{~mL})$ and $\mathrm{H}_{2} \mathrm{O}(5 \mathrm{~mL})$ at $0{ }^{\circ} \mathrm{C}$ was added dropwise $\mathrm{NaOCl}(5.2 \mathrm{~mL}, 10.76 \mathrm{mmol})$. After the reaction mixture was stirred at $0{ }^{\circ} \mathrm{C}$ for 1 $\mathrm{h}, \mathrm{NaHSO}_{3}(5 \%, 10 \mathrm{~mL})$ was added. Then, the mixture was extracted with $\mathrm{CH}_{2} \mathrm{Cl}_{2}$, washed with $\mathrm{H}_{2} \mathrm{O}$, dried $\left(\mathrm{Na}_{2} \mathrm{SO}_{4}\right)$ and filtered. The filtrate was concentrated under reduced pressure to give 7 as a white solid: $R_{\mathrm{f}}=0.70\left(3: 1\right.$, hexane/EtOAc); $m p$ 98-100 ${ }^{\circ} \mathrm{C} ;[\alpha]_{\mathrm{D}}{ }^{27}=+9.4(0.01$, $\mathrm{CH}_{2} \mathrm{Cl}_{2}$ ); IR (KBr) 3483 (br), 2957, 2880, 1709, 1461, $1338 \mathrm{~cm}^{-1} ;{ }^{1} \mathrm{H}$ NMR (200 MHz, $\left.\mathrm{CDCl}_{3}\right): \delta 10.07(\mathrm{~s}, 1 \mathrm{H}), 3.56(\mathrm{br} \mathrm{s}, 1 \mathrm{H}), 2.23-1.17(\mathrm{~m}, 2 \mathrm{H}), 1.92-1.30(\mathrm{~m}, 6 \mathrm{H}), 1.10(\mathrm{~s}, 3 \mathrm{H})$, $0.99(\mathrm{~s}, 3 \mathrm{H}) ;{ }^{13} \mathrm{C}$ NMR $\left(50 \mathrm{MHz}, \mathrm{CDCl}_{3}\right): \delta 207.4,84.0,49.9,48.9,48.6,37.3,25.6,24.0$, 22.4, 21.5. HRMS calcd for $\mathrm{C}_{10} \mathrm{H}_{16} \mathrm{O}_{2}$ : 168.1150; found: 168.1155 . 

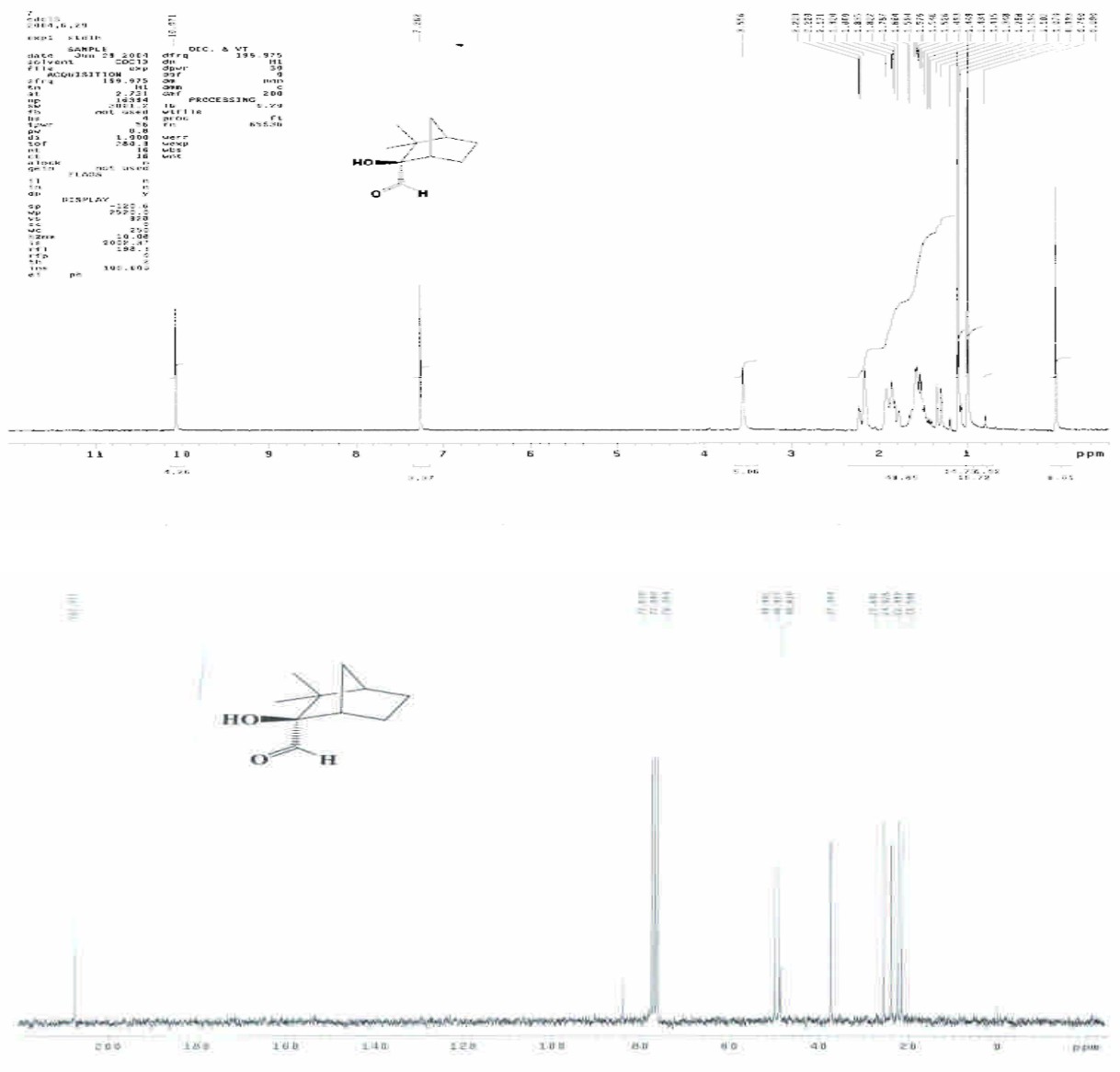

General procedure for the reaction of carbinol 6 with an alkyl or aryl lithium reagent

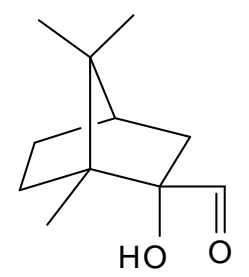

6
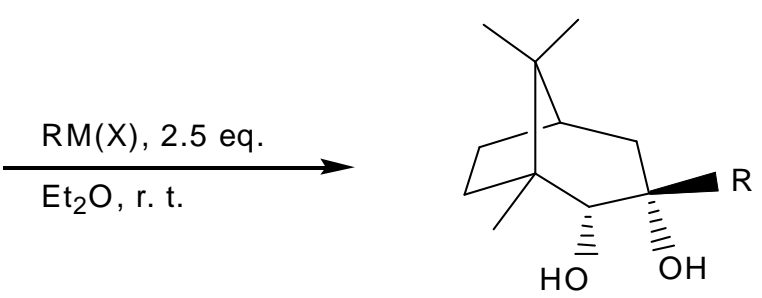

To a solution of carbinol $6(0.45 \mathrm{~g}, 2.47 \mathrm{mmol})$ in diethyl ether $(10 \mathrm{~mL})$ was added dropwise an alkyl or aryl lithium reagent $(6.18 \mathrm{mmol})$ in solvent(s). The reaction mixture was stirred at room temperature for $30 \mathrm{~min}$, then quenched with aqueous $\mathrm{NH}_{4} \mathrm{Cl}$ and extracted with ethyl acetate $(3 \times 10 \mathrm{~mL})$. The organic layers were combined and washed with water, dried $\left(\mathrm{Na}_{2} \mathrm{SO}_{4}\right)$ and concentrated under reduced pressure. The residue was purified with flash column chromatography (6:1, hexane/EtOAc) to give the corresponding alkyl/aryl[3.2.1]bicyclic diol. 
<smiles>CC1(C)C2CCC(C2)C1(O)C=O</smiles>

7

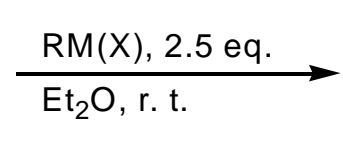

$\mathrm{Et}_{2} \mathrm{O}, \mathrm{r}$.<smiles></smiles>

$10(\mathrm{R}=\mathrm{Me})$

To a solution of carbinol $7(0.50 \mathrm{~g}, 2.98 \mathrm{mmol})$ in diethyl ether $(10 \mathrm{~mL})$ was added dropwise a Grignard or lithium reagent $(7.45 \mathrm{mmol})$. The reaction mixture was stirred at room temperature for $30 \mathrm{~min}$, then quenched with aqueous $\mathrm{NH}_{4} \mathrm{Cl}$ and extracted with ethyl acetate $(3 \mathrm{x} 10 \mathrm{~mL})$. The organic layers were combined and washed with water, dried $\left(\mathrm{Na}_{2} \mathrm{SO}_{4}\right)$ and concentrated under reduced pressure. The residue was purified with flash column chromatography $(6: 1$, hexane/EtOAc) to give the corresponding [3.2.1]bicyclic diol.

General procedure for the ring-cleavage of bicyclic diols 10, 14, 16, 17 and 21.

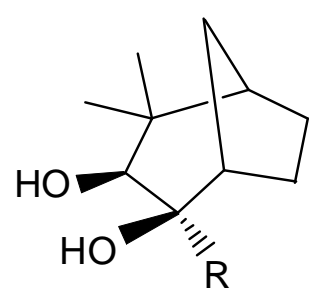

10: $\mathrm{R}=\mathrm{Me}$

14: $\mathrm{R}=\mathrm{Et}$

16: $\mathrm{R}=$ Allyl

17: $\mathrm{R}=n-\mathrm{Bu}$

21: $\mathrm{R}=\mathrm{Ph}$

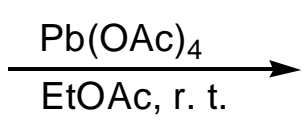

24 (94\%): R = Me

$25(90 \%): \mathrm{R}=\mathrm{Et}$

26 (80 \%): R = Allyl

$27(81 \%): \mathrm{R}=n$-Bu

$28(89 \%): \mathrm{R}=\mathrm{Ph}$

A solution of [3.2.1]bicyclic diol $(0.43 \mathrm{mmol})$ in ethyl acetate $(5 \mathrm{~mL})$ was treated with lead tetraacetate $(0.56 \mathrm{mmol})$. The reaction mixture was stirred at room temperature for $5 \mathrm{~min}$, then quenched with water $(10 \mathrm{~mL})$ and extracted with ethyl acetate $(3 \times 10 \mathrm{~mL})$. The organic layers were combined and washed with water, dried $\left(\mathrm{Na}_{2} \mathrm{SO}_{4}\right)$ and concentrated under reduced pressure. The residue was purified with flash column chromatography (3:1, hexane/EtOAc) to give the corresponding cyclopentane derivative. 
[3.2.1]bicyclic diol $11^{2}$, a solid: $\mathrm{R}_{\mathrm{f}}=0.18\left(6: 1\right.$, hexane/EtOAc); $\mathrm{mp} 87-88^{\circ} \mathrm{C}$;

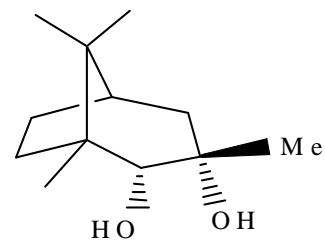

$[\alpha]_{\mathrm{D}}{ }^{25}=+5.9\left(0.10, \mathrm{CH}_{2} \mathrm{Cl}_{2}\right) ; \mathrm{IR}(\mathrm{KBr}): 3361(\mathrm{br}), 2966,1366,1026, \mathrm{~cm}^{-1} ;{ }^{1} \mathrm{H}$ NMR $(200 \mathrm{MHz}$, $\mathrm{CDCl}_{3}$ ): $\delta 3.37$ (dd, J=1.4, 7.2 Hz, 1H), 2.39 (dd, J=3.6, 7.2 Hz, OH), 2.27 (s, OH), 2.01-1.60 (m, $7 \mathrm{H}), 1.22(\mathrm{~s}, 3 \mathrm{H}), 0.92(\mathrm{~s}, 3 \mathrm{H}), 0.88(\mathrm{~s}, 3 \mathrm{H}), 0.86(\mathrm{~s}, 3 \mathrm{H}) ;{ }^{13} \mathrm{C} \mathrm{NMR}\left(50 \mathrm{MHz}, \mathrm{CDCl}_{3}\right): \delta 77.6$, 70.4, 46.7, 44.5, 44.3, 41.5, 30.9, 27.8, 26.0, 24.6, 18.8, 16.9. Anal Calcd. for $\mathrm{C}_{12} \mathrm{H}_{22} \mathrm{O}_{2}: \mathrm{C}$, 72.68; H, 11.18. Found: C, 72.30; H, 11.25.

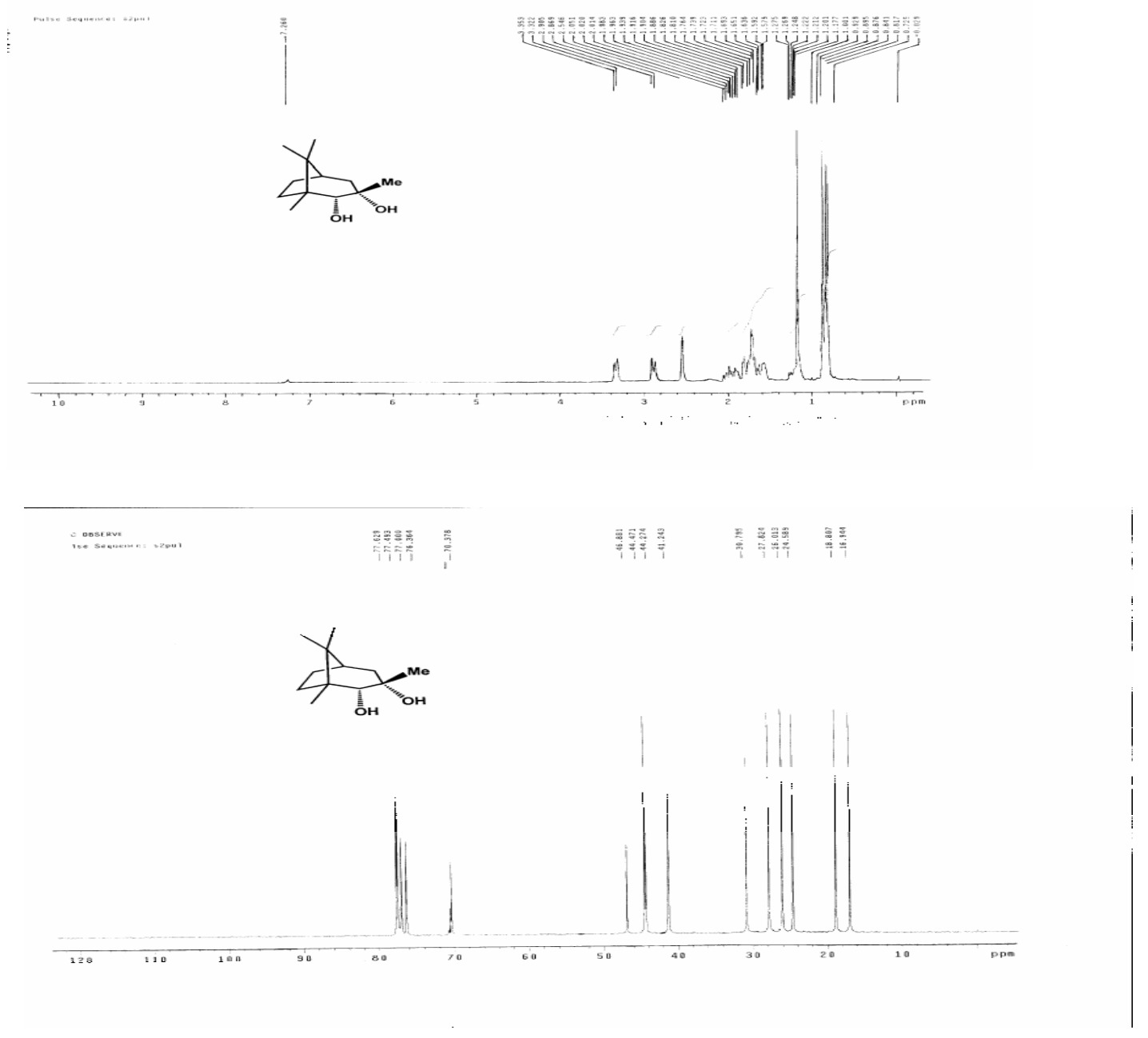


[3.2.1]bicyclic diol 12, a solid: $\mathrm{R}_{\mathrm{f}}=0.41\left(6: 1\right.$, hexane/EtOAc); $\mathrm{mp} 74-76^{\circ} \mathrm{C}$;

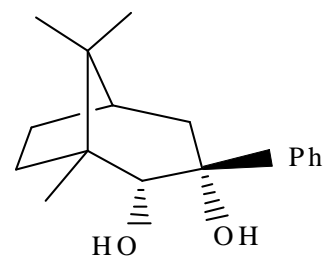

$[\alpha]_{\mathrm{D}}{ }^{27}=+2.1(0.01, \mathrm{EtOH}) ; \mathrm{IR}(\mathrm{KBr}): 3336(\mathrm{br}), 3059,2964,1980,1860,1496,1445 \mathrm{~cm}^{-1} ;{ }^{1} \mathrm{H}$ NMR (200 MHz, $\left.\mathrm{CDCl}_{3}\right): \delta$ 7.54-7.18 (m, 5H), $3.91(\mathrm{~s}, 1 \mathrm{H}), 2.49$ (br s, $\left.2 \mathrm{OH}\right), 2.21-1.71(\mathrm{~m}, 6 \mathrm{H})$, 1.39-1.26 (m, 1H), $1.11(\mathrm{~s}, 3 \mathrm{H}), 0.95(\mathrm{~s}, 6 \mathrm{H}) ;{ }^{13} \mathrm{C} \mathrm{NMR}\left(50 \mathrm{MHz}, \mathrm{CDCl}_{3}\right): \delta$ 149.2, 128.1, 126.6, 124.8, 78.0, 74.7, 47.3, 44.9, 44.5, 42.2, 28.5, 26.1, 25.0, 18.9, 16.9. Anal Calcd. for $\mathrm{C}_{17} \mathrm{H}_{24} \mathrm{O}_{2}$ : C, 78.42; H, 9.29. Found: C, 78.35; H, 9.25.
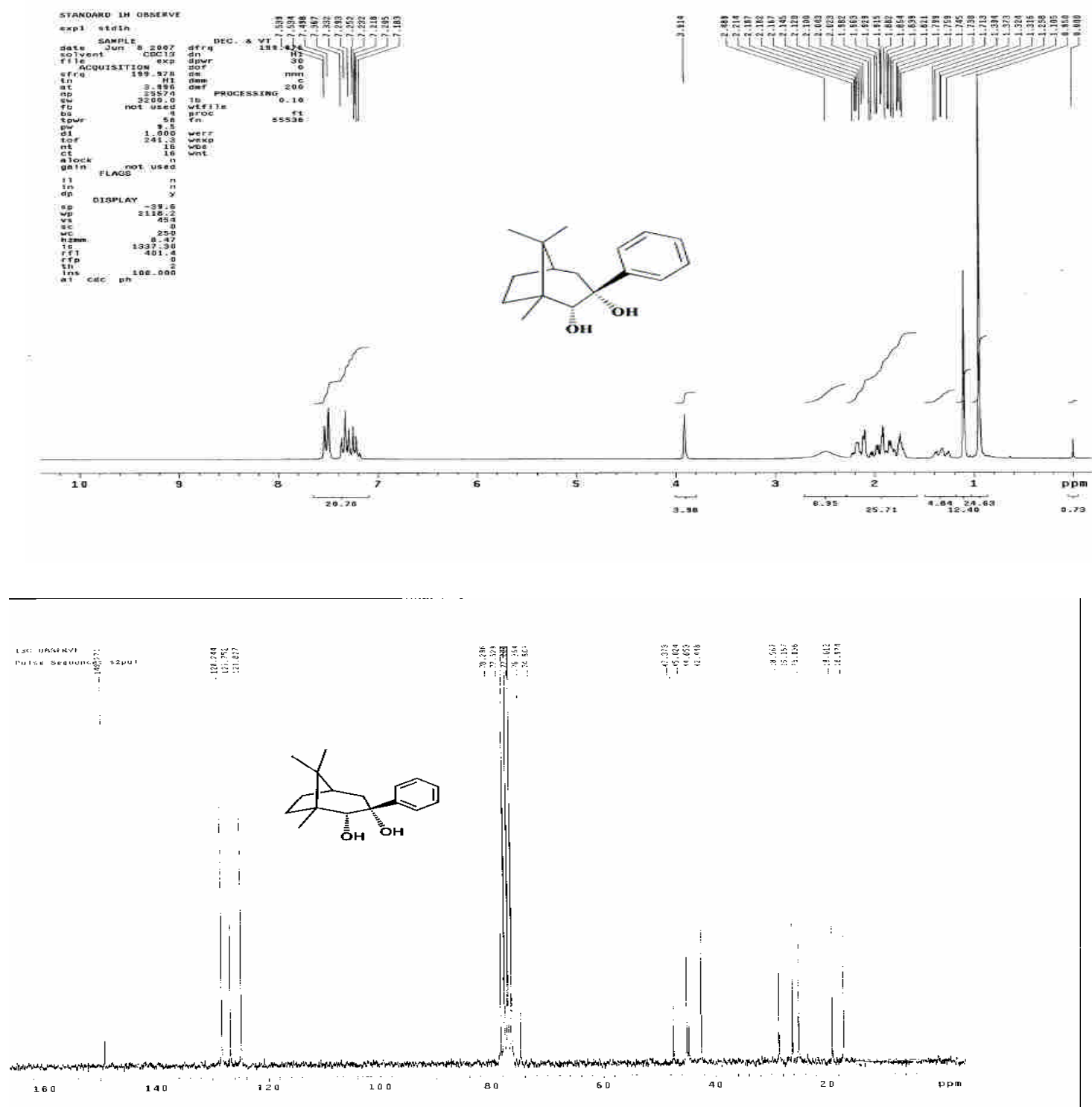
[3.2.1]bicyclic diol 13, a solid: $\mathrm{R}_{\mathrm{f}}=0.30$ (6:1, hexane/EtOAc); mp 100-102 ${ }^{\circ} \mathrm{C}$;

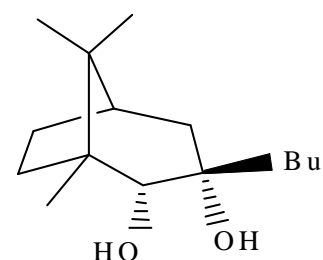

$[\alpha]_{\mathrm{D}}{ }^{25}=+11.3\left(0.05, \mathrm{CH}_{2} \mathrm{Cl}_{2}\right) ; \mathrm{IR}(\mathrm{KBr}): 3357(\mathrm{br}), 1449,1390,1035 \mathrm{~cm}^{-1} ;{ }^{1} \mathrm{H}$ NMR $(200 \mathrm{MHz}$, $\left.\mathrm{CDCl}_{3}\right): \delta 3.37(\mathrm{~d}, \mathrm{~J}=7.0 \mathrm{~Hz}, 1 \mathrm{H}), 2.46(\mathrm{~d}, \mathrm{~J}=7.0 \mathrm{~Hz}, \mathrm{OH}), 2.25(\mathrm{~s}, 1 \mathrm{H}), 2.09-1.95(\mathrm{~m}, 1 \mathrm{H}), 1.87$ -1.56 (m, 6H), 1.46-1.17 (m, 6H), $0.91(\mathrm{t}, \mathrm{J}=5.4 \mathrm{~Hz}, 3 \mathrm{H}), 0.91(\mathrm{~s}, 3 \mathrm{H}), 0.87(\mathrm{~s}, 3 \mathrm{H}), 0.84(\mathrm{~s}, 3 \mathrm{H})$; ${ }^{13} \mathrm{C}$ NMR $\left(50 \mathrm{MHz}, \mathrm{CDCl}_{3}\right): \delta 77.5,72.2,47.1,44.4,44.1,43.5,39.2,27.9,26.2,25.2,24.7,23.3$, 18.9, 17.0, 14.1. Anal. Calcd for $\mathrm{C}_{15} \mathrm{H}_{28} \mathrm{O}_{2}:$ C, 74.95; H, 11.74. Found: C, 75.00; H, 11.84.
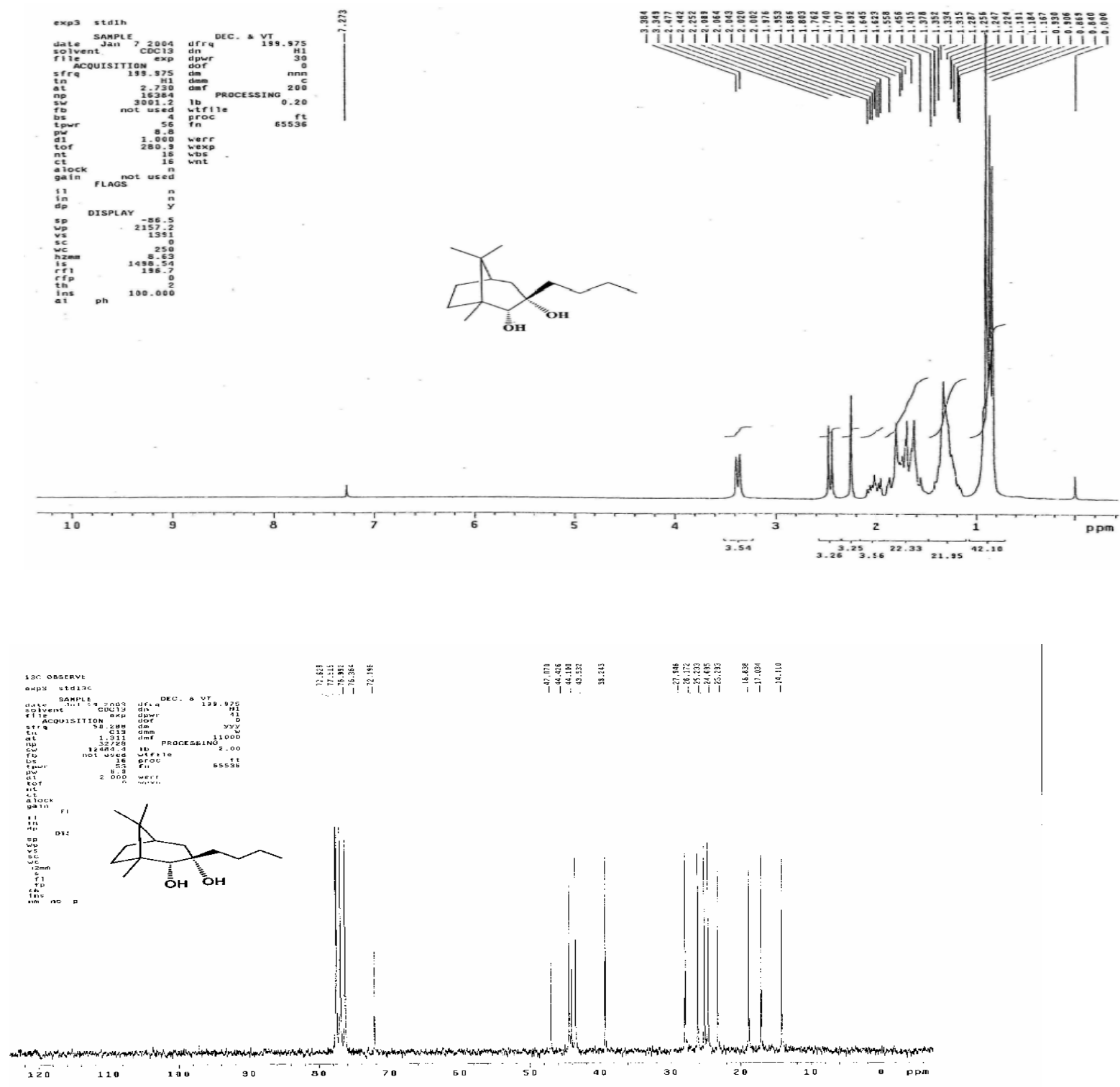
[3.2.1]bicyclic diol 10, a solid: $\mathrm{R}_{\mathrm{f}}=0.55$ (3:1, hexane/EtOAc); $\mathrm{mp} 137-138{ }^{\circ} \mathrm{C}$;

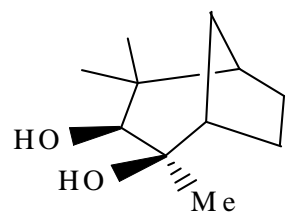

$[\alpha]_{\mathrm{D}}{ }^{27}=+23.5\left(0.01, \mathrm{CH}_{2} \mathrm{Cl}_{2}\right)$; IR(KBr): $3364(\mathrm{br}), 2935,2866,1660,1630,1454,1142 \mathrm{~cm}^{-1} ;{ }^{1} \mathrm{H}$ NMR (200 MHz, $\left.\mathrm{CDCl}_{3}\right): \delta 3.03(\mathrm{~d}, \mathrm{~J}=8.8 \mathrm{~Hz}, 1 \mathrm{H}), 2.30(\mathrm{~m}, 1 \mathrm{H}), 2.06(\mathrm{~m}, 1 \mathrm{H}), 1.83-1.35(\mathrm{~m}$, $7 \mathrm{H}), 1.23(\mathrm{~s}, 3 \mathrm{H}), 1.17(\mathrm{~m}, 1 \mathrm{H}), 1.04(\mathrm{~s}, 3 \mathrm{H}), 0.96(\mathrm{~s}, 3 \mathrm{H}) ;{ }^{13} \mathrm{C} \mathrm{NMR}\left(50 \mathrm{MHz}, \mathrm{CDCl}_{3}\right): \delta 76.6$, 75.6, 46.6, 46.5, 39.2, 29.4, 27.1, 26.1, 25.9, 24.3, 21.1. Anal Calcd. for $\mathrm{C}_{11} \mathrm{H}_{20} \mathrm{O}_{2}: \mathrm{C}, 71.70 ; \mathrm{H}$, 10.94. Found: C, 71.33; H, 11.19.
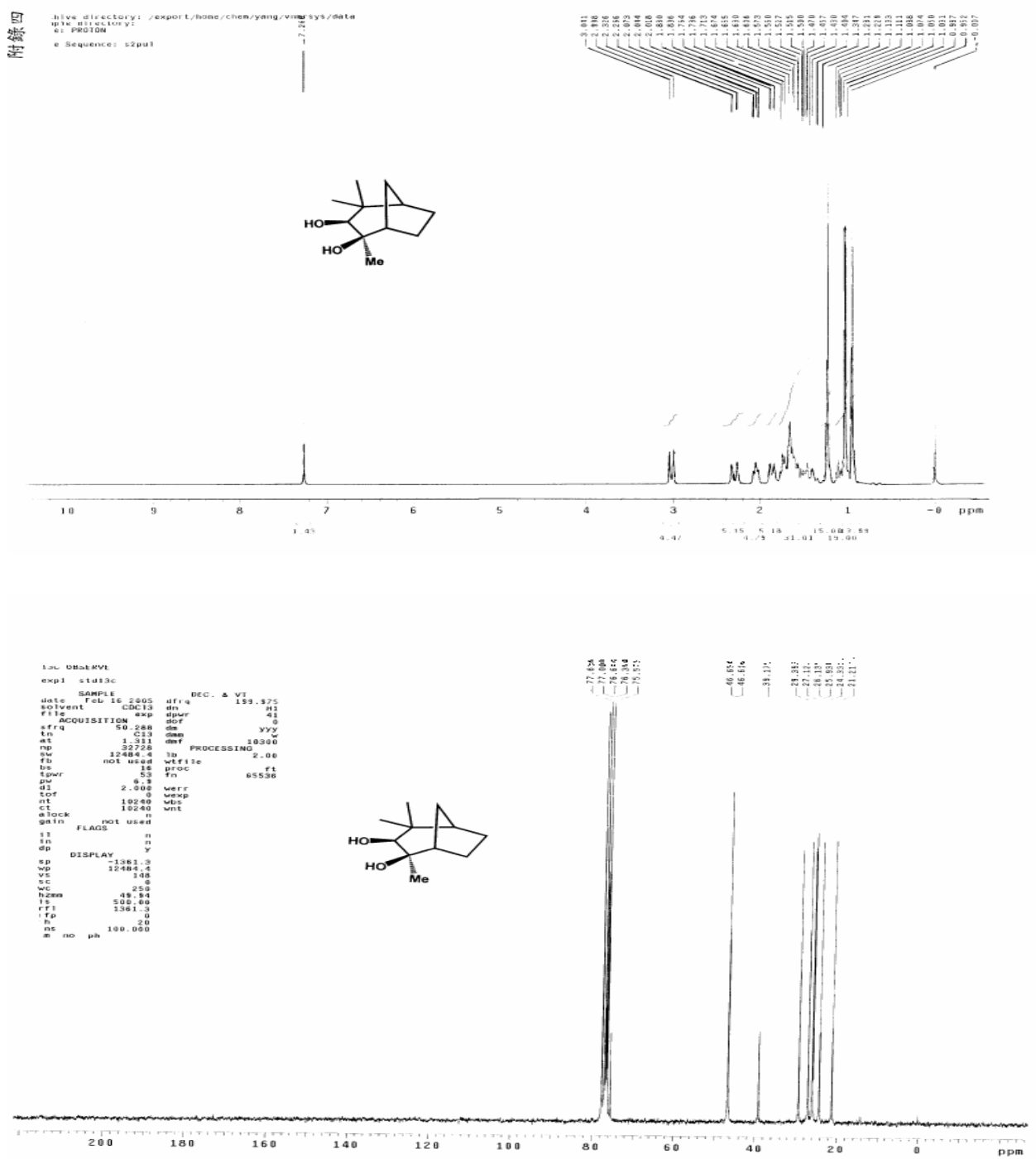
[3.2.1]bicyclic diol 14, a solid: $\mathrm{R}_{\mathrm{f}}=0.45$ (6:1, hexane/EtOAc); $\mathrm{mp} 161-162{ }^{\circ} \mathrm{C}$;

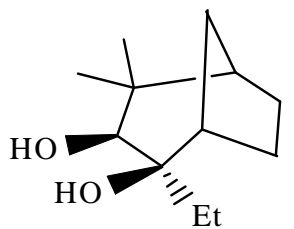

$[\alpha]_{\mathrm{D}}{ }^{27}=+21.0\left(0.01, \mathrm{CH}_{2} \mathrm{Cl}_{2}\right) ; \mathrm{IR}(\mathrm{KBr}): 3338(\mathrm{br}), 2956,2864,1461,1362 \mathrm{~cm}^{-1} ;{ }^{1} \mathrm{H}$ NMR $(200$ $\left.\mathrm{MHz}, \mathrm{CDCl}_{3}\right): \delta 3.00(\mathrm{~d}, \mathrm{~J}=8.2 \mathrm{~Hz}, 1 \mathrm{H}), 2.26-2.21(\mathrm{~m}, 2 \mathrm{H}), 1.87-1.37(\mathrm{~m}, 9 \mathrm{H}), 1.13(\mathrm{~m}, 1 \mathrm{H})$, $1.05(\mathrm{~s}, 3 \mathrm{H}), 0.96(\mathrm{~s}, 3 \mathrm{H}), 0.87(\mathrm{t}, \mathrm{J}=7.6 \mathrm{~Hz}, 3 \mathrm{H}) ;{ }^{13} \mathrm{C} \mathrm{NMR}\left(50 \mathrm{MHz}, \mathrm{CDCl}_{3}\right): \delta 77.4,76.8,46.6$, 40.8, 39.2. 29.2, 29.0, 27.2, 25.1, 24.3, 21.5. 6.7. Anal Calcd. for $\mathrm{C}_{12} \mathrm{H}_{22} \mathrm{O}_{2}: \mathrm{C}, 72.68 ; \mathrm{H}, 11.18$. Found: C, 72.62; H, 10.80 .
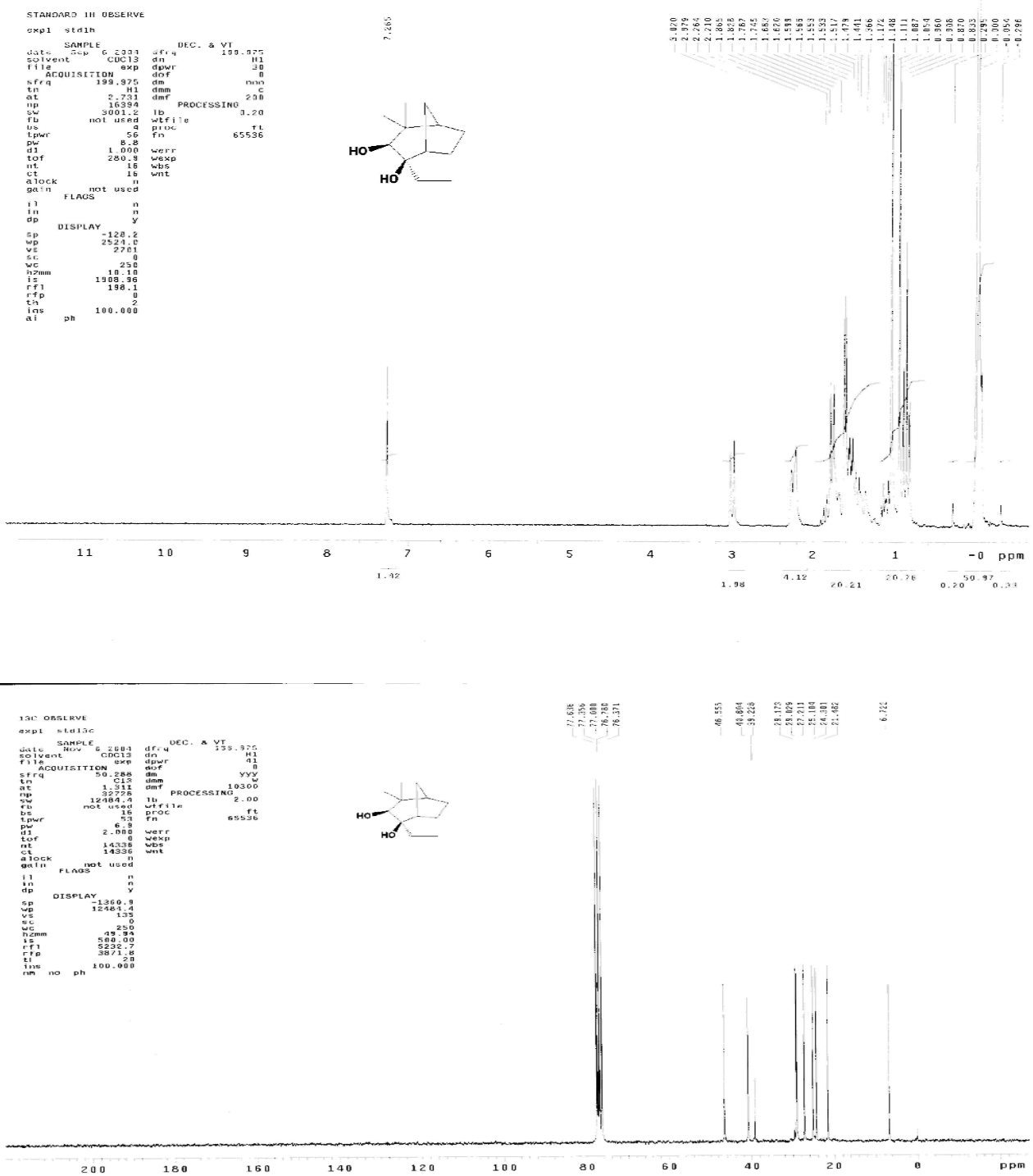
[3.2.1]bicyclic diol 15, a solid: $\mathrm{R}_{\mathrm{f}}=0.54$ (6:1, hexane/EtOAc); $\mathrm{mp} 115-116{ }^{\circ} \mathrm{C}$;

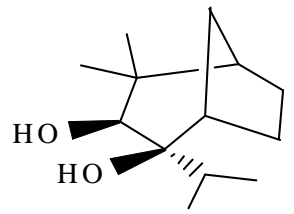

$[\alpha]_{\mathrm{D}}{ }^{27}=+16.1\left(0.01, \mathrm{CH}_{2} \mathrm{Cl}_{2}\right) ; \mathrm{IR}(\mathrm{KBr}): 3452(\mathrm{br}), 2957,2873,1468,1364 \mathrm{~cm}^{-1} ;{ }^{1} \mathrm{H} \mathrm{NMR}(200$ $\left.\mathrm{MHz}, \mathrm{CDCl}_{3}\right): \delta 3.24(\mathrm{~d}, \mathrm{~J}=8.0 \mathrm{~Hz}, 1 \mathrm{H}), 2.32-2.21(\mathrm{~m}, 2 \mathrm{H}), 1.97(\mathrm{~m}, 1 \mathrm{H}), 1.94-1.45(\mathrm{~m}, 7 \mathrm{H})$, $1.14(\mathrm{~m}, 1 \mathrm{H}), 1.06(\mathrm{~d}, \mathrm{~J}=6.8 \mathrm{~Hz}, 3 \mathrm{H}), 1.05(\mathrm{~s}, 3 \mathrm{H}), 0.97$ (s, 3H), 0.92 (d, J=6.8 Hz, 3H); ${ }^{13} \mathrm{C} \mathrm{NMR}\left(50 \mathrm{MHz}, \mathrm{CDCl}_{3}\right): \delta 76.5,76.4,46.6,42.0,39.8,35.4,29.8,27.3,24.8,24.2,22.2$, 18.6. Anal Calcd. for $\mathrm{C}_{13} \mathrm{H}_{24} \mathrm{O}_{2}$ : C, 73.54; $\mathrm{H}, 11.39$. Found: C, 73.42; H, 11.09.
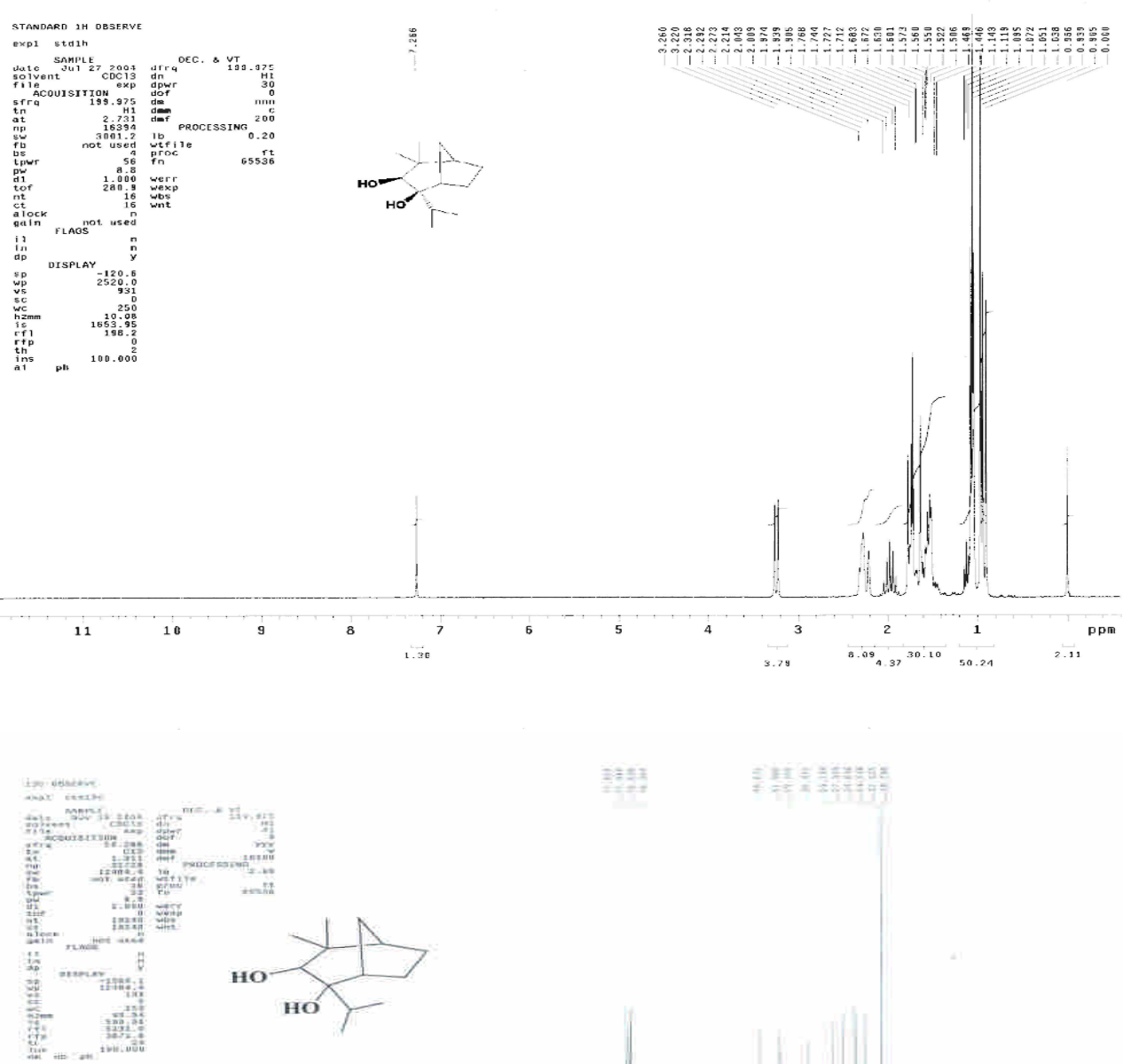
[3.2.1]bicyclic diol 16, a solid: $\mathrm{R}_{\mathrm{f}}=0.54$ (3:1, hexane/EtOAc); $\mathrm{mp} 154-155{ }^{\circ} \mathrm{C}$;

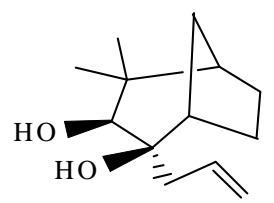

$[\alpha]_{\mathrm{D}}{ }^{27}=+13.0\left(0.01, \mathrm{CH}_{2} \mathrm{Cl}_{2}\right) ; \mathrm{IR}(\mathrm{KBr}): 3341(\mathrm{br}), 3069,2960,2914,2868,1639,1455,1439$ $\mathrm{cm}^{-1}$; ${ }^{1} \mathrm{H}$ NMR $\left(200 \mathrm{MHz}, \mathrm{CDCl}_{3}\right): \delta 5.93(\mathrm{~m}, 1 \mathrm{H}), 5.22-5.09(\mathrm{~m}, 2 \mathrm{H}), 3.03(\mathrm{~d}, \mathrm{~J}=9.2 \mathrm{~Hz}, 1 \mathrm{H})$, $2.53(\mathrm{dd}, \mathrm{J}=8.6,13.8 \mathrm{~Hz}, 1 \mathrm{H}), 2.33-2.24(\mathrm{~m}, 2 \mathrm{H}), 2.13(\mathrm{t}, \mathrm{J}=5.2 \mathrm{~Hz}, 1 \mathrm{H}), 1.90(\mathrm{~d}, \mathrm{~J}=9.2 \mathrm{~Hz}, 1 \mathrm{H})$, $1.89(\mathrm{~s}, 1 \mathrm{H}), 1.77-1.45(\mathrm{~m}, 5 \mathrm{H}), 1.15-1.06(\mathrm{~m}, 1 \mathrm{H}), 1.04(\mathrm{~s}, 3 \mathrm{H}), 0.97(\mathrm{~s}, 3 \mathrm{H})$;

${ }^{13} \mathrm{C} \mathrm{NMR}\left(50 \mathrm{MHz}, \mathrm{CDCl}_{3}\right): \delta 133.9,119.5,76.5,46.5,43.1,41.5,39.4,29.2,27.2,25.3,24.3$, 21.4. Anal Calcd. for $\mathrm{C}_{13} \mathrm{H}_{22} \mathrm{O}_{2}: \mathrm{C}, 74.24 ; \mathrm{H}, 10.54$. Found: $\mathrm{C}, 74.44 ; \mathrm{H}, 10.37$.
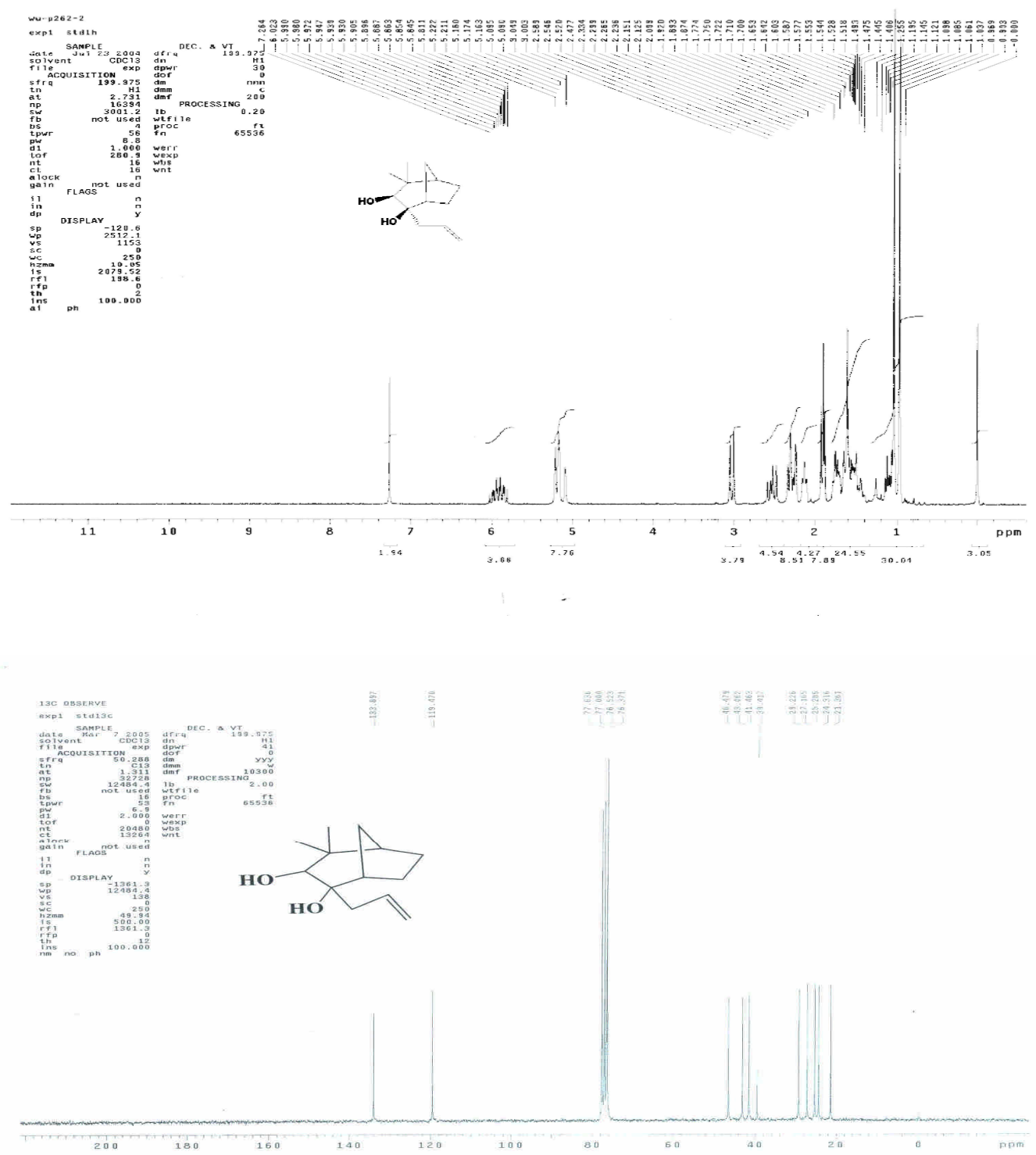
[3.2.1]bicyclic diol 17, a solid: $\mathrm{R}_{\mathrm{f}}=0.53$ (3:1, hexane/EtOAc); mp 146-147 ${ }^{\circ} \mathrm{C}$;

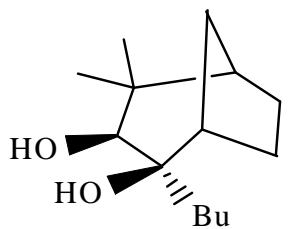

$[\alpha]_{\mathrm{D}}{ }^{27}=+17.0\left(0.01, \mathrm{CH}_{2} \mathrm{Cl}_{2}\right) ; \mathrm{IR}(\mathrm{KBr}): 3351(\mathrm{br}), 2957,2860,1460,1305 \mathrm{~cm}^{-1} ;{ }^{1} \mathrm{H}$ NMR (200 $\left.\mathrm{MHz}, \mathrm{CDCl}_{3}\right): \delta 2.99(\mathrm{~d}, \mathrm{~J}=8.1 \mathrm{~Hz}, 1 \mathrm{H}), 2.26-2.21(\mathrm{~m}, 2 \mathrm{H}), 1.84(\mathrm{~d}, \mathrm{~J}=8.1 \mathrm{~Hz}, 1 \mathrm{H}), 1.74-1.25(\mathrm{~m}$, 12H), $1.13(\mathrm{~m}, 1 \mathrm{H}), 1.04(\mathrm{~s}, 3 \mathrm{H}), 0.95(\mathrm{~s}, 3 \mathrm{H}), 0.91(\mathrm{t}, \mathrm{J}=6.6 \mathrm{~Hz}, 3 \mathrm{H}) ; \quad{ }^{13} \mathrm{C} \mathrm{NMR}(50 \mathrm{MHz}$, $\left.\mathrm{CDCl}_{3}\right): \delta 77.4,76.8,46.6,41.6,39.2,36.7,29.2,27.2,25.2,24.6,24.3,23.3,21.4,14.0$. Anal Calcd. for $\mathrm{C}_{14} \mathrm{H}_{26} \mathrm{O}_{2}$ : C, 74.29; H, 11.58. Found: C, 74.32; H, 11.38.
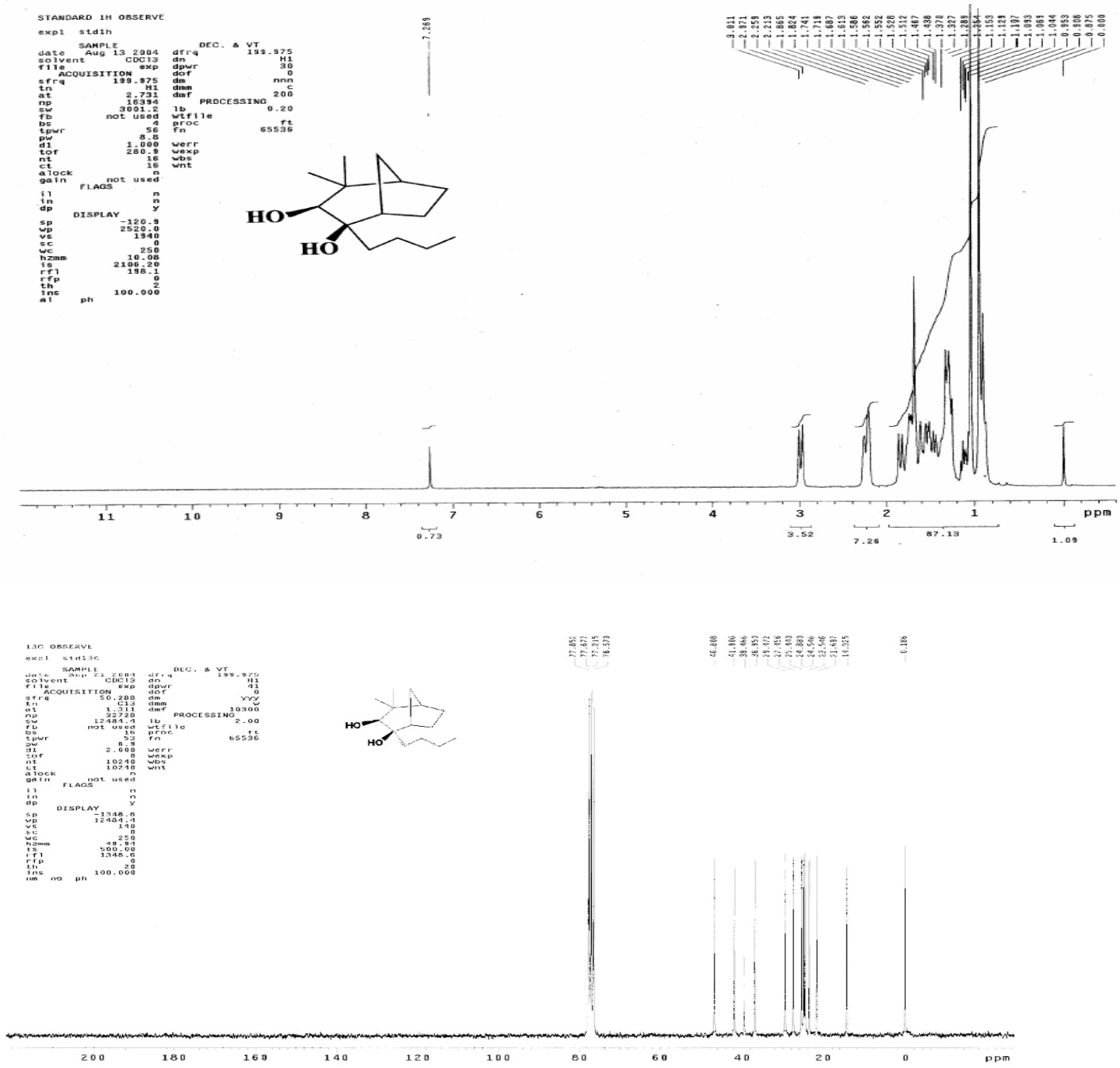
[3.2.1]bicyclic diol 18, a solid: $\mathrm{R}_{\mathrm{f}}=0.46\left(6: 1\right.$, hexane/EtOAc); $\mathrm{mp} 120-121{ }^{\circ} \mathrm{C}$;

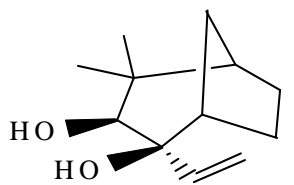

$[\alpha]_{\mathrm{D}}{ }^{21}=+17.5\left(0.01, \mathrm{CH}_{2} \mathrm{Cl}_{2}\right) ; \mathrm{IR}(\mathrm{KBr}): 3322(\mathrm{br}), 3050,2955,2862,1634,1360 \mathrm{~cm}^{-1} ;{ }^{1} \mathrm{H} \mathrm{NMR}$ $\left(200 \mathrm{MHz}, \mathrm{CDCl}_{3}\right): \delta 6.04(\mathrm{dd}, \mathrm{J}=10.8,17.4 \mathrm{~Hz}, 1 \mathrm{H}), 5.33(\mathrm{dd}, \mathrm{J}=1.4,17.4 \mathrm{~Hz}, 1 \mathrm{H}), 5.20$ (dd, $\mathrm{J}=1.4,10.8 \mathrm{~Hz}, 1 \mathrm{H}), 3.24(\mathrm{~d}, \mathrm{~J}=6.2 \mathrm{~Hz}, 1 \mathrm{H}), 2.39$ (d, J=12.0 Hz, 1H), 2.15 (d, J=11.2 Hz, 1H), $2.10(\mathrm{~s}, 1 \mathrm{H}), 1.75(\mathrm{~m}, 1 \mathrm{H}), 1.67-1.50(\mathrm{~m}, 5 \mathrm{H}), 1.16(\mathrm{~m}, 1 \mathrm{H}), 1.09(\mathrm{~s}, 3 \mathrm{H}), 1.00(\mathrm{~s}, 3 \mathrm{H}) ;{ }^{13} \mathrm{C} \mathrm{NMR}$ $\left(50 \mathrm{MHz}, \mathrm{CDCl}_{3}\right): \delta 142.9,114.4,77.35,74.47,46.65,45.08,38.86,29.41,27.23,25.07,24.35$, 21.38. HRMS Calcd. for $\mathrm{C}_{12} \mathrm{H}_{20} \mathrm{O}_{2}$ : 196.1463; found: 196.1460 .
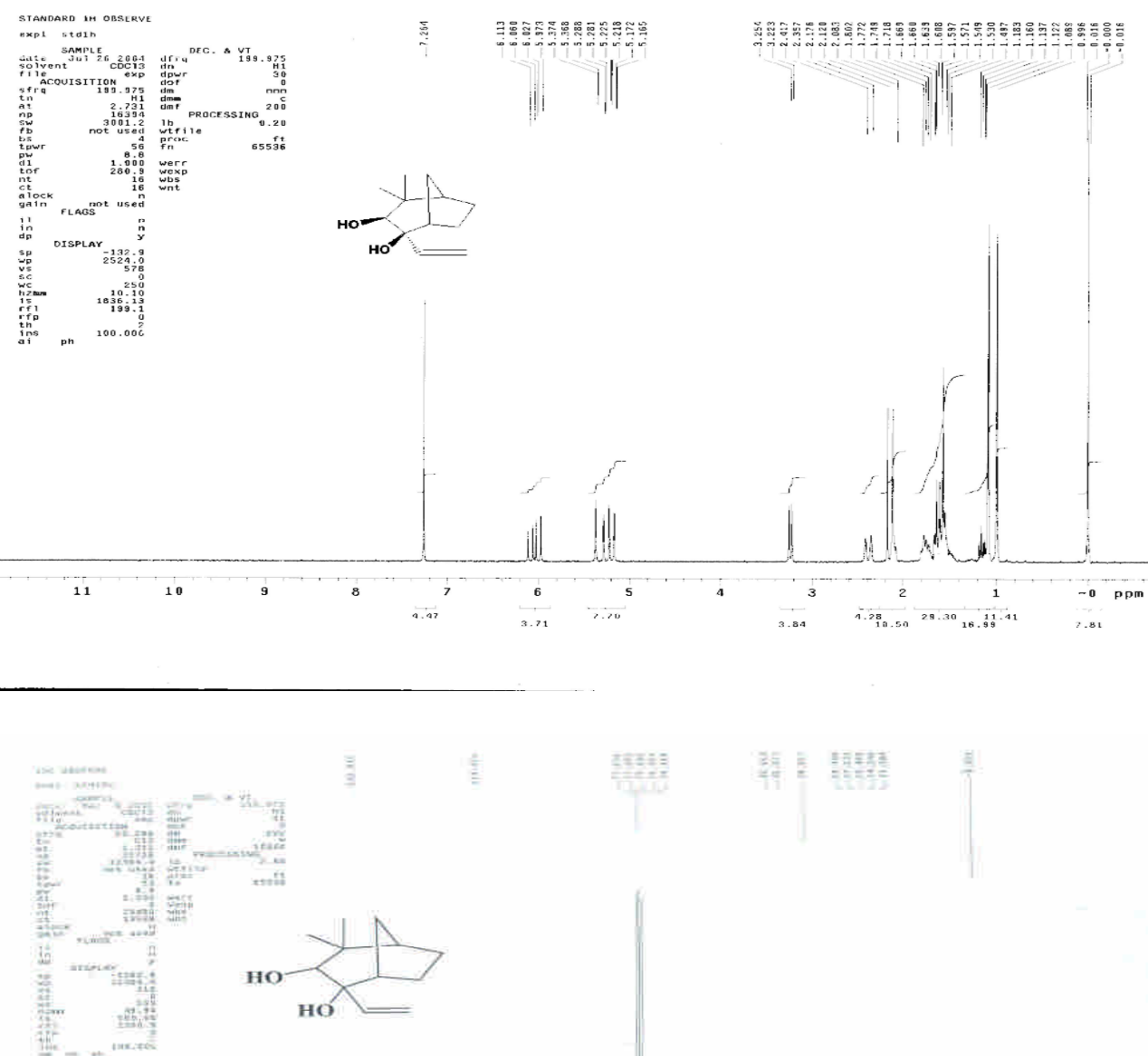
[3.2.1]bicyclic diol 19, a solid: $\mathrm{R}_{\mathrm{f}}=0.14$ (3:1, hexane/EtOAc); $\mathrm{mp} 161-162{ }^{\circ} \mathrm{C}$;

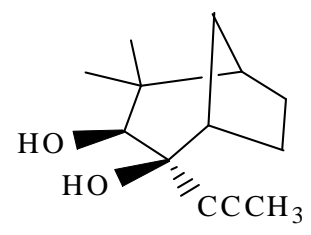

$[\alpha]_{\mathrm{D}}{ }^{27}=+11.5\left(0.01, \mathrm{CH}_{2} \mathrm{Cl}_{2}\right) ; \mathrm{IR}(\mathrm{KBr}): 3306(\mathrm{br}), 2915,2361,1306 \mathrm{~cm}^{-1} ;{ }^{1} \mathrm{H}$ NMR $(200 \mathrm{MHz}$, $\left.\mathrm{CDCl}_{3}\right): \delta 3.31(\mathrm{~d}, \mathrm{~J}=6.2 \mathrm{~Hz}, 1 \mathrm{H}), 2.71(\mathrm{br} \mathrm{s}, \mathrm{OH}), 2.36-2.24(\mathrm{~m}, 2 \mathrm{H}), 2.12(\mathrm{~d}, \mathrm{~J}=6.2 \mathrm{~Hz}, \mathrm{OH})$, $1.84(\mathrm{~s}, 3 \mathrm{H}), 1.72-1.49(\mathrm{~m}, 5 \mathrm{H}), 1.14(\mathrm{~m}, 1 \mathrm{H}), 1.02(\mathrm{~s}, 3 \mathrm{H}), 0.97(\mathrm{~s}, 3 \mathrm{H}) ;{ }^{13} \mathrm{C}$ NMR $(50 \mathrm{MHz}$, $\left.\mathrm{CDCl}_{3}\right): \delta 82.9,78.7,76.9,74.5,46.3,46.1,38.8,28.6,27.1,26.0,24.2,21.0,3.6 . \quad$ Anal Calcd. for $\mathrm{C}_{13} \mathrm{H}_{20} \mathrm{O}_{2}$ : C, 74.96; $\mathrm{H}, 9.68$. Found: C, 74.98; H, 9.64.
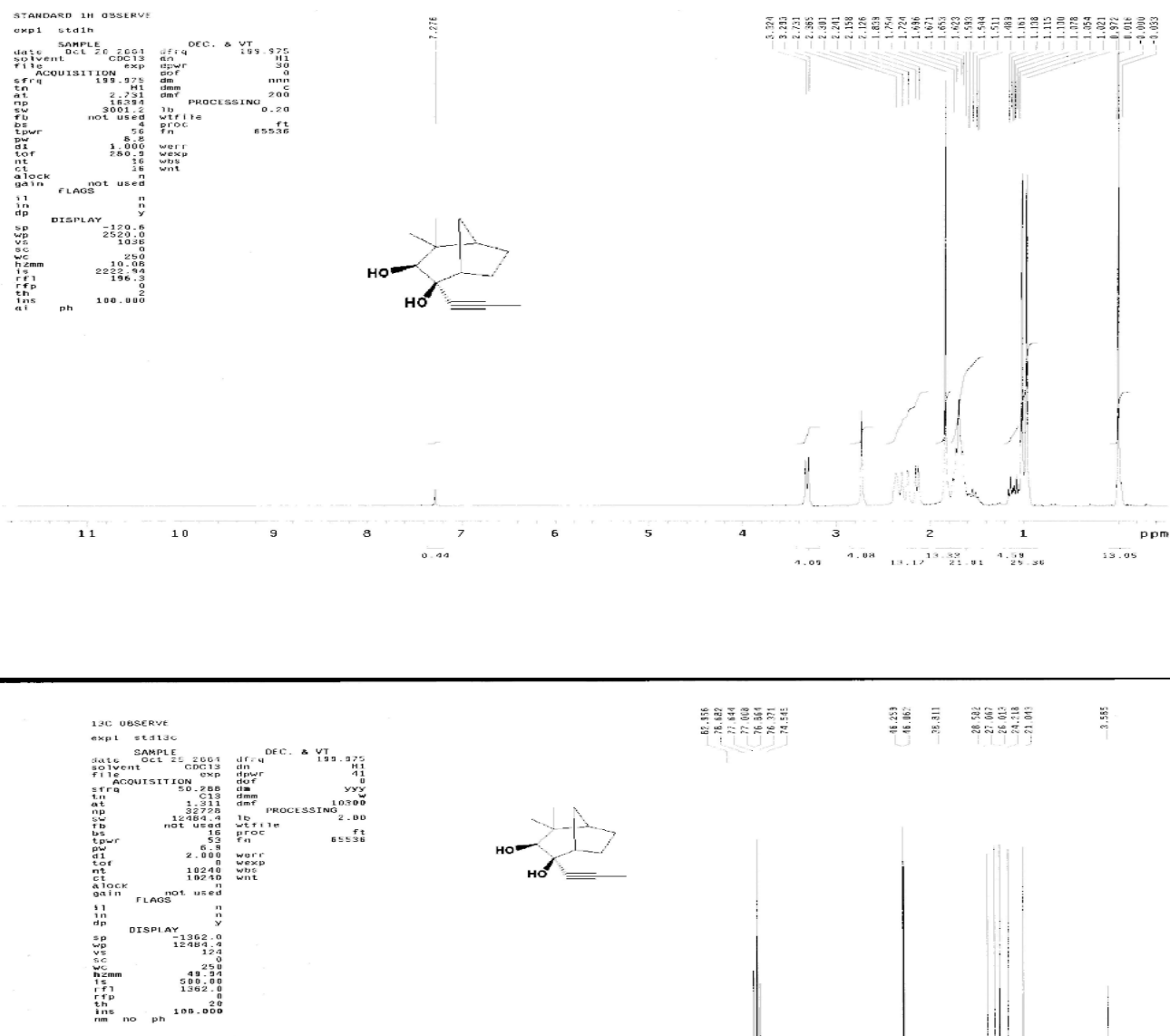
[3.2.1]bicyclic diol 20, a solid: $\mathrm{R}_{\mathrm{f}}=0.68$ (3:1, hexane/EtOAc); $\mathrm{mp} 126-127^{\circ} \mathrm{C}$;

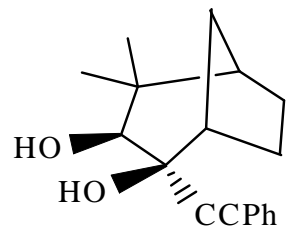

$[\alpha]_{\mathrm{D}}{ }^{27}=+12.5\left(0.01, \mathrm{CH}_{2} \mathrm{Cl}_{2}\right) ; \mathrm{IR}(\mathrm{KBr}): 3445,3058,2960,2363,1950,1905,1820,1653,1490$, $1405 \mathrm{~cm}^{-1} ;{ }^{1} \mathrm{H}$ NMR $\left(200 \mathrm{MHz}, \mathrm{CDCl}_{3}\right): \delta 7.45-7.28(\mathrm{~m}, 5 \mathrm{H}), 3.46(\mathrm{~s}, 1 \mathrm{H}), 2.51(\mathrm{~s}, 1 \mathrm{H}), 2.34(\mathrm{~d}$, $\mathrm{J}=12.0 \mathrm{~Hz}, 1 \mathrm{H}), 2.30-2.01(\mathrm{~m}, 3 \mathrm{H}), 1.80-1.53(\mathrm{~m}, 4 \mathrm{H}), 1.54(\mathrm{~m}, 1 \mathrm{H}), 1.07,(\mathrm{~s}, 3 \mathrm{H}), 1.01(\mathrm{~s}, 3 \mathrm{H})$; ${ }^{13} \mathrm{C} \mathrm{NMR}\left(50 \mathrm{MHz}, \mathrm{CDCl}_{3}\right.$ ): $\delta 131.7,128.3,128.2,122.6,92.8,82.8,76.8,75.1,46.3,46.0,39.0$, 28.7, 27.2, 26.1, 24.3, 21.1. HRMS calcd for $\mathrm{C}_{18} \mathrm{H}_{22} \mathrm{O}_{2}$ : 270.1620; found: 270.1624.

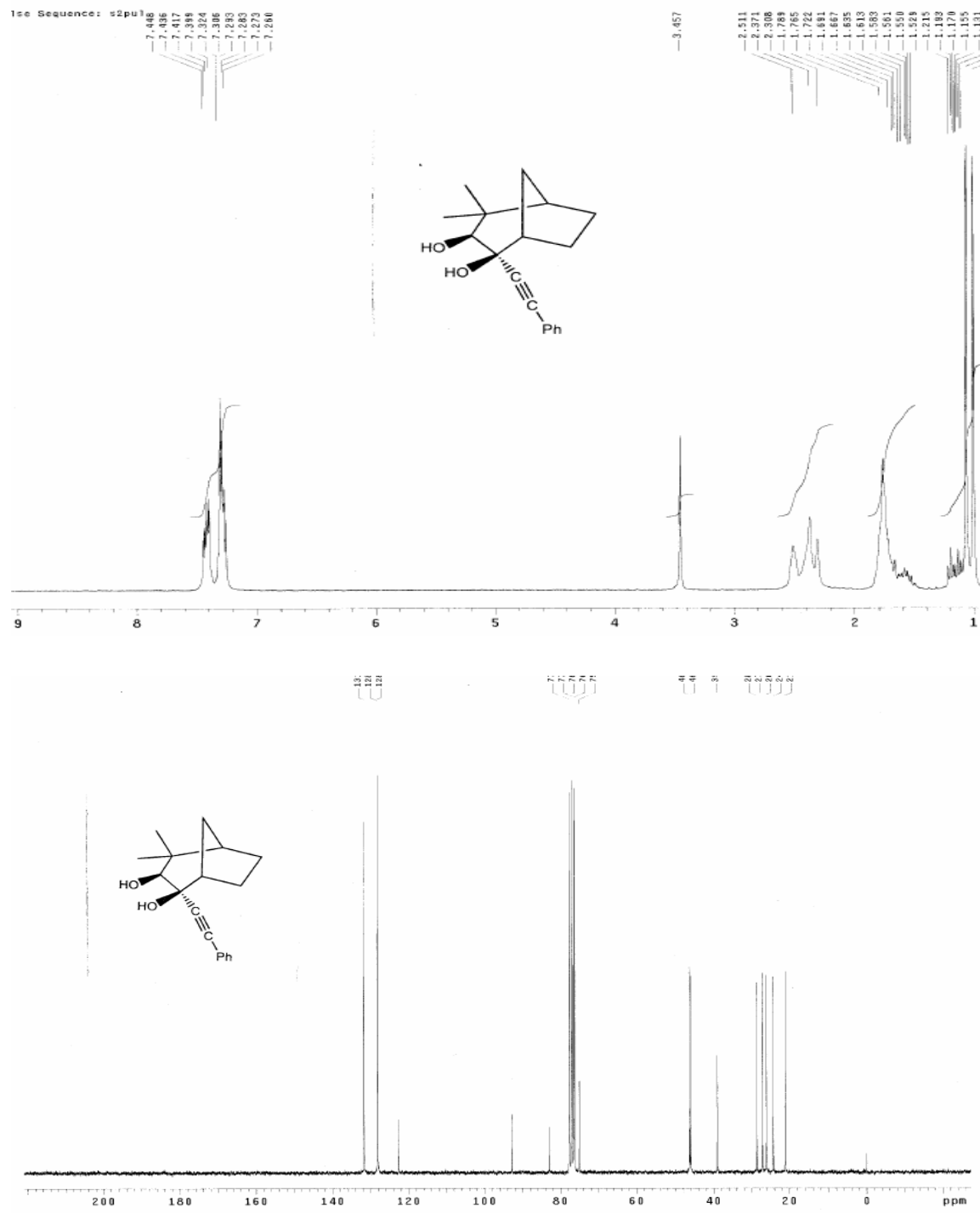


[3.2.1]bicyclic diol 21, a solid: $\mathrm{R}_{\mathrm{f}}=0.62$ (3:1, hexane/EtOAc); mp 169-170 ${ }^{\circ} \mathrm{C}$;

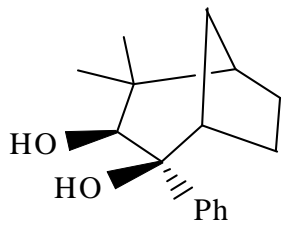

$[\alpha]_{\mathrm{D}}^{27}=+10.5\left(0.01, \mathrm{CH}_{2} \mathrm{Cl}_{2}\right) ; \mathrm{IR}(\mathrm{KBr}): 3420(\mathrm{br}), 3060,2960,1910,1860,1636,1446,1304$ $\mathrm{cm}^{-1} ;{ }^{1} \mathrm{H}$ NMR $\left(200 \mathrm{MHz}, \mathrm{CDCl}_{3}\right): \delta 7.56-7.21(\mathrm{~m}, 5 \mathrm{H}), 3.98(\mathrm{~d}, \mathrm{~J}=6.2 \mathrm{~Hz}, 1 \mathrm{H}), 2.59(\mathrm{~s}, 1 \mathrm{H})$, $2.53(\mathrm{~d}, \mathrm{~J}=12.2 \mathrm{~Hz}, 1 \mathrm{H}), 2.32(\mathrm{t}, \mathrm{J}=5.1 \mathrm{~Hz}, 1 \mathrm{H}), 1.85-1.19(\mathrm{~m}, 7 \mathrm{H}), 1.16(\mathrm{~s}, 3 \mathrm{H}), 1.08(\mathrm{~s}, 3 \mathrm{H}) ;{ }^{13} \mathrm{C}$ NMR (50 MHz, $\left.\mathrm{CDCl}_{3}\right): \delta 146.2,128.2,127.0,126.4,78.9,72.4,47.5,46.7,39.0,29.8,27.4$, 24.7, 24.2, 21.6. Anal Calcd. for $\mathrm{C}_{16} \mathrm{H}_{22} \mathrm{O}_{2}$ : C, 78.01; H, 9.00. Found: C, 77.61; H, 8.75.

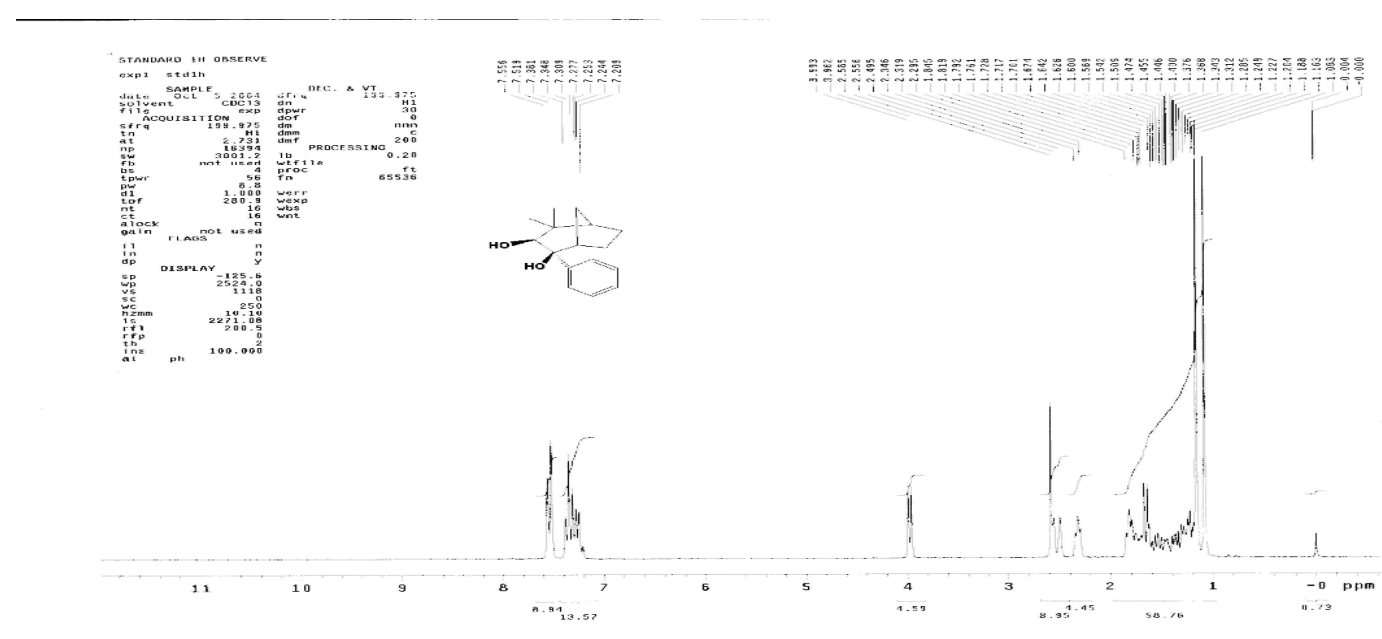

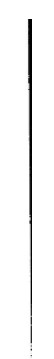

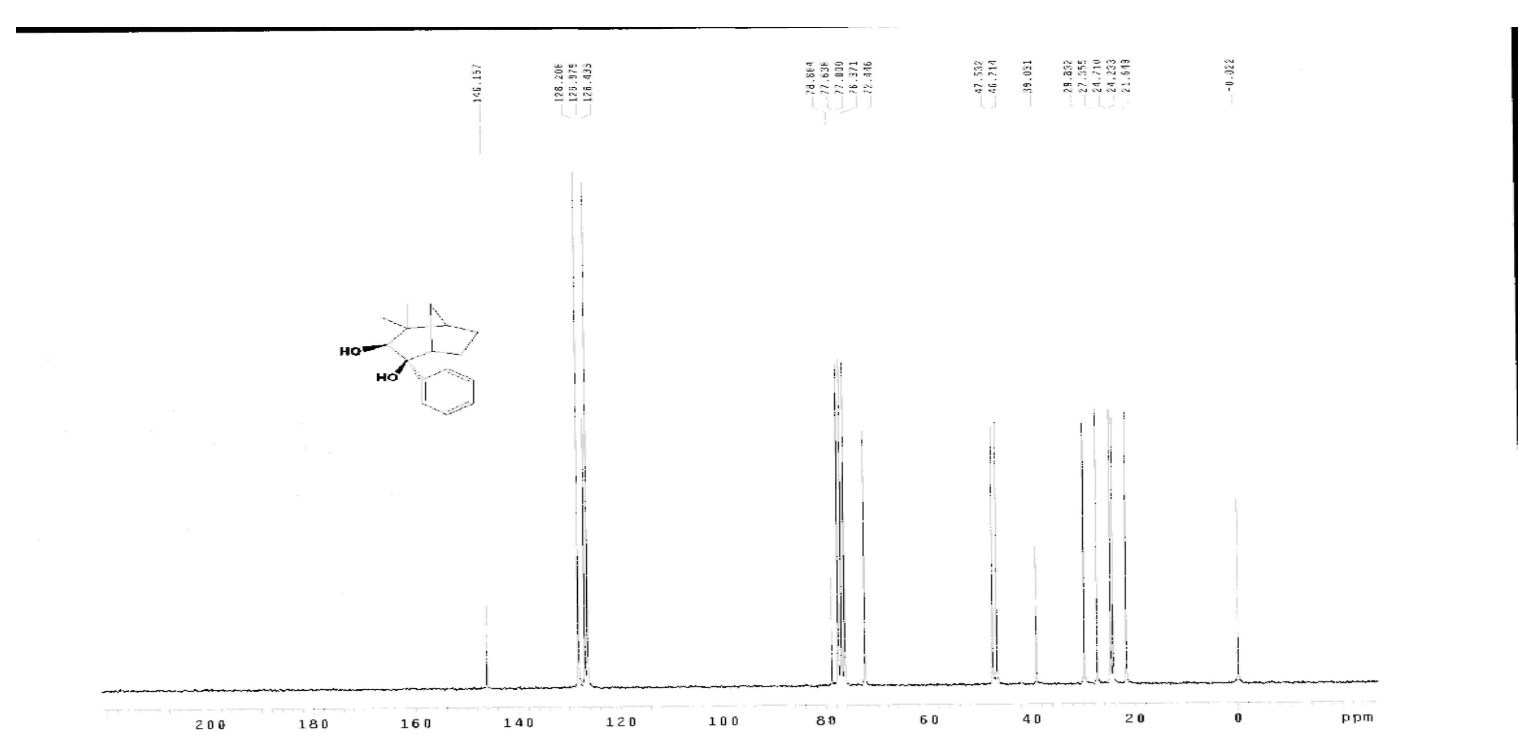


[3.2.1]bicyclic diol 22, a solid: $\mathrm{R}_{\mathrm{f}}=0.56$ (3:1, hexane/EtOAc); mp 143-144 ${ }^{\circ} \mathrm{C}$;

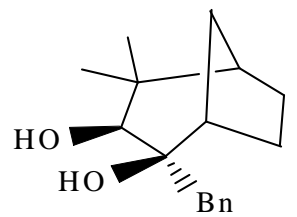

$[\alpha]_{\mathrm{D}}{ }^{27}=+2.5\left(0.01, \mathrm{CH}_{2} \mathrm{Cl}_{2}\right) ; \mathrm{IR}(\mathrm{KBr}): 3841(\mathrm{br}), 3062,3028,2961,2873,1920,1850,1711$, 1603, 1453, $1308 \mathrm{~cm}^{-1} ;{ }^{1} \mathrm{H}$ NMR (200 MHz, $\left.\mathrm{CDCl}_{3}\right): \delta$ 7.36-7.16 (m, 5H), $3.13(\mathrm{~d}, \mathrm{~J}=9.4 \mathrm{~Hz}, 1 \mathrm{H})$, $3.11(\mathrm{~d}, \mathrm{~J}=13.6 \mathrm{~Hz}, 1 \mathrm{H}), 2.79$ (d, J=13.6 Hz, 1H), $2.19(\mathrm{~m}, 1 \mathrm{H}), 2.11(\mathrm{~d}, \mathrm{~J}=9.4 \mathrm{~Hz}, 1 \mathrm{H}), 1.93(\mathrm{~m}$, 1H), $1.77(\mathrm{~s}, 1 \mathrm{H}), 1.75-1.49(\mathrm{~m}, 5 \mathrm{H}), 1.05(\mathrm{~s}, 3 \mathrm{H}), 1.03(\mathrm{~m}, 1 \mathrm{H}), 1.00(\mathrm{~s}, 3 \mathrm{H}) ;{ }^{13} \mathrm{C}$ NMR $(50 \mathrm{MHz}$, $\left.\mathrm{CDCl}_{3}\right): \delta 136.9,130.7,128.3,126.5,76.8,76.6,46.7,42.2,39.5,29.0,27.2,25.7,24.3,21.4$. Anal Calcd. for $\mathrm{C}_{17} \mathrm{H}_{24} \mathrm{O}_{2}$ : C, 78.42; H, 9.29. Found: C, 78.15; H, 8.90.
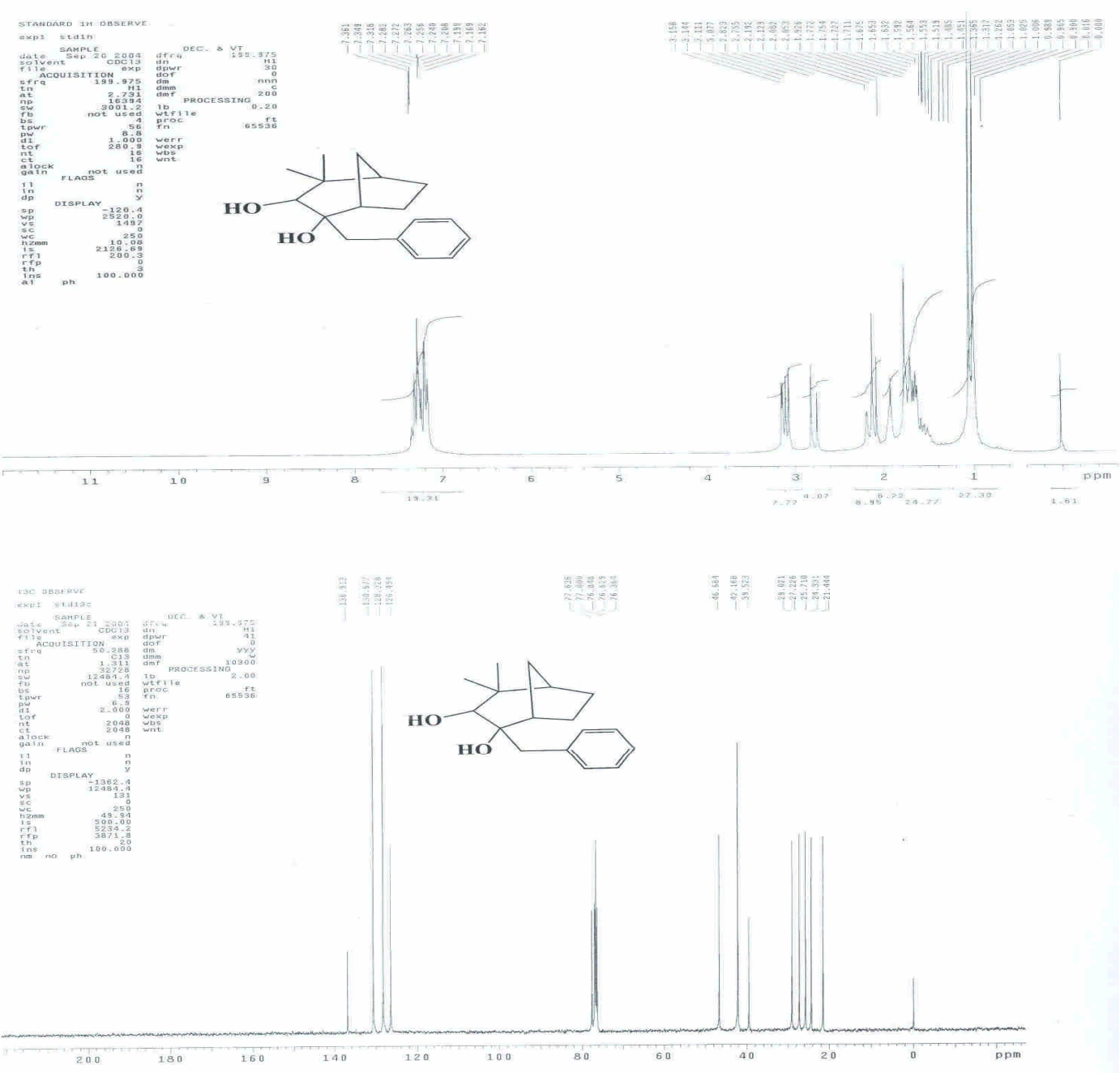
[3.2.1]bicyclic diol 23, a solid: $\mathrm{R}_{\mathrm{f}}=0.18$ (3:1, hexane/EtOAc); mp $125-126{ }^{\circ} \mathrm{C}$;

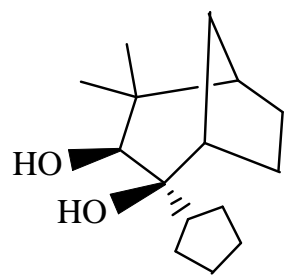

$[\alpha]_{\mathrm{D}}{ }^{27}=+18.6\left(0.01, \mathrm{CH}_{2} \mathrm{Cl}_{2}\right) ; \mathrm{IR}(\mathrm{KBr}): 3503(\mathrm{br}), 2950,2868,1452,1362,1308 \mathrm{~cm}^{-1} ;{ }^{1} \mathrm{H} \mathrm{NMR}$ $\left(200 \mathrm{MHz}, \mathrm{CDCl}_{3}\right): \delta 3.16(\mathrm{~d}, \mathrm{~J}=7.6 \mathrm{~Hz}, 1 \mathrm{H}), 2.30-2.22(\mathrm{~m}, 2 \mathrm{H}), 2.13-1.99(\mathrm{~m}, 1 \mathrm{H}), 1.85(\mathrm{~s}, 1 \mathrm{H})$, 1.79-1.26 (m, $14 \mathrm{H}), 1.08(\mathrm{~m}, 1 \mathrm{H}), 1.04(\mathrm{~s}, 3 \mathrm{H}), 0.95(\mathrm{~s}, 3 \mathrm{H}) ;{ }^{13} \mathrm{C}$ NMR $\left(50 \mathrm{MHz}, \mathrm{CDCl}_{3}\right): \delta 78.0$, 76.4, 48.5, 46.5, 44.0, 39.5, 29.5, 28.0, 27.2, 26.7, 25.8, 24.8, 24.2, 23.6, 22.0. Anal Calcd. for $\mathrm{C}_{15} \mathrm{H}_{26} \mathrm{O}_{2}: \mathrm{C}, 75.58 ; \mathrm{H}, 10.99$. Found: $\mathrm{C}, 75.69 ; \mathrm{H}, 10.78$.

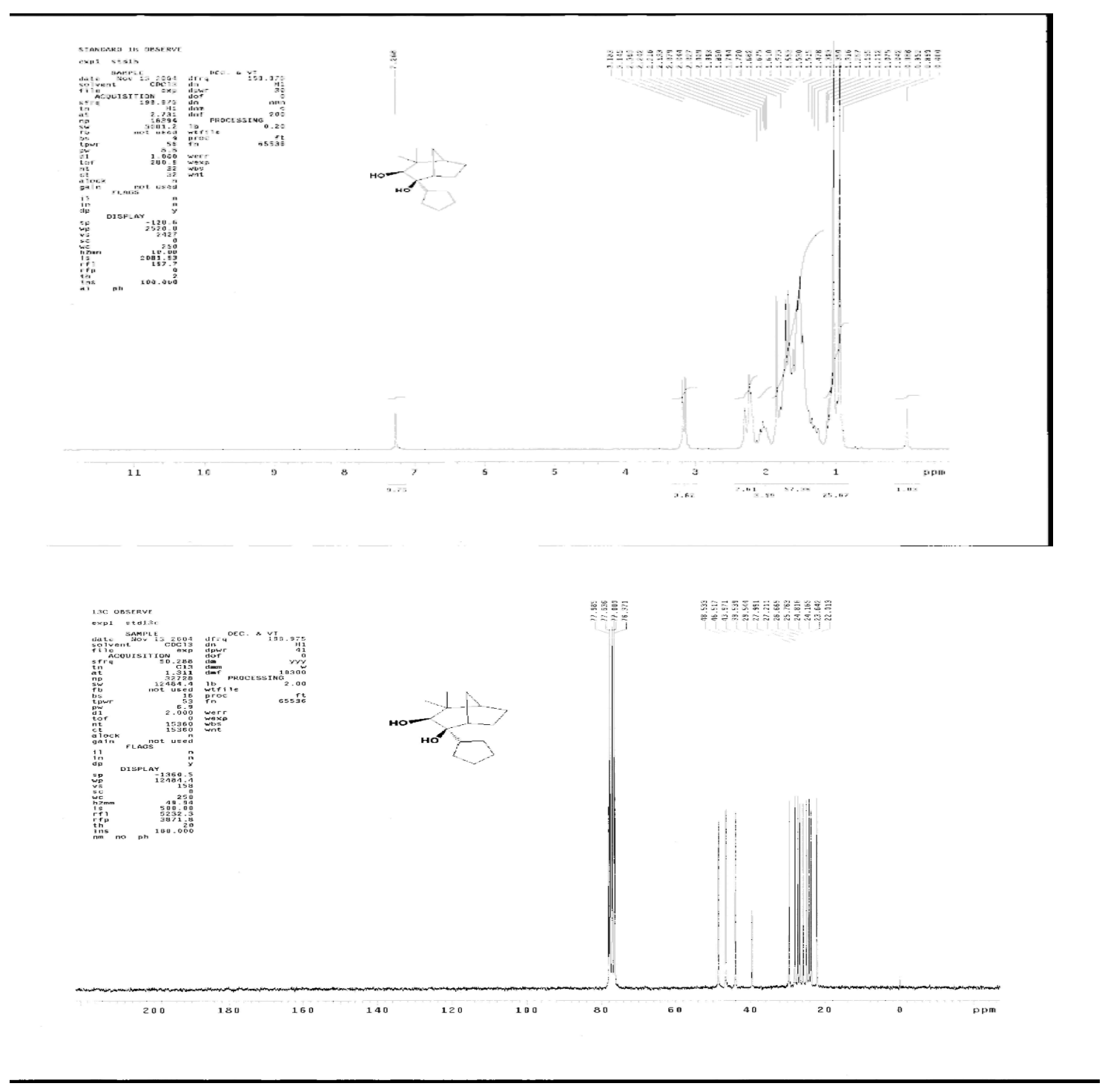


Cyclopentane derivative 24, a syrup: $\mathrm{R}_{\mathrm{f}}=0.55$ (3:1, hexane/EtOAc);<smiles>CC(=O)[C@@H]1CC[C@H](C(C)(C)C=O)C1</smiles>

$[\alpha]_{\mathrm{D}}{ }^{27}=+1.9\left(0.01, \mathrm{CH}_{2} \mathrm{Cl}_{2}\right)$; IR(film): 2963, 2873, 2701, 2349, 1724, 1468, 1402, $1365 \mathrm{~cm}^{-1} ;{ }^{1} \mathrm{H}$ NMR $\left(200 \mathrm{MHz}, \mathrm{CDCl}_{3}\right): \delta 9.50(\mathrm{~s}, 1 \mathrm{H}), 2.88(\mathrm{~m}, 1 \mathrm{H}), 2.14(\mathrm{~s}, 3 \mathrm{H}), 2.09-1.14(\mathrm{~m}, 7 \mathrm{H}), 1.03(\mathrm{~s}$, $6 \mathrm{H}) ;{ }^{13} \mathrm{C} \mathrm{NMR}\left(50 \mathrm{MHz}, \mathrm{CDCl}_{3}\right): \delta 210.3,206.0,51.3,47.3,45.2,29.7,28.8,27.3,25.9,19.2$, 19.0. Anal Calcd. for $\mathrm{C}_{11} \mathrm{H}_{18} \mathrm{O}_{2}:$ 182.1307. Found : 182.1303 .
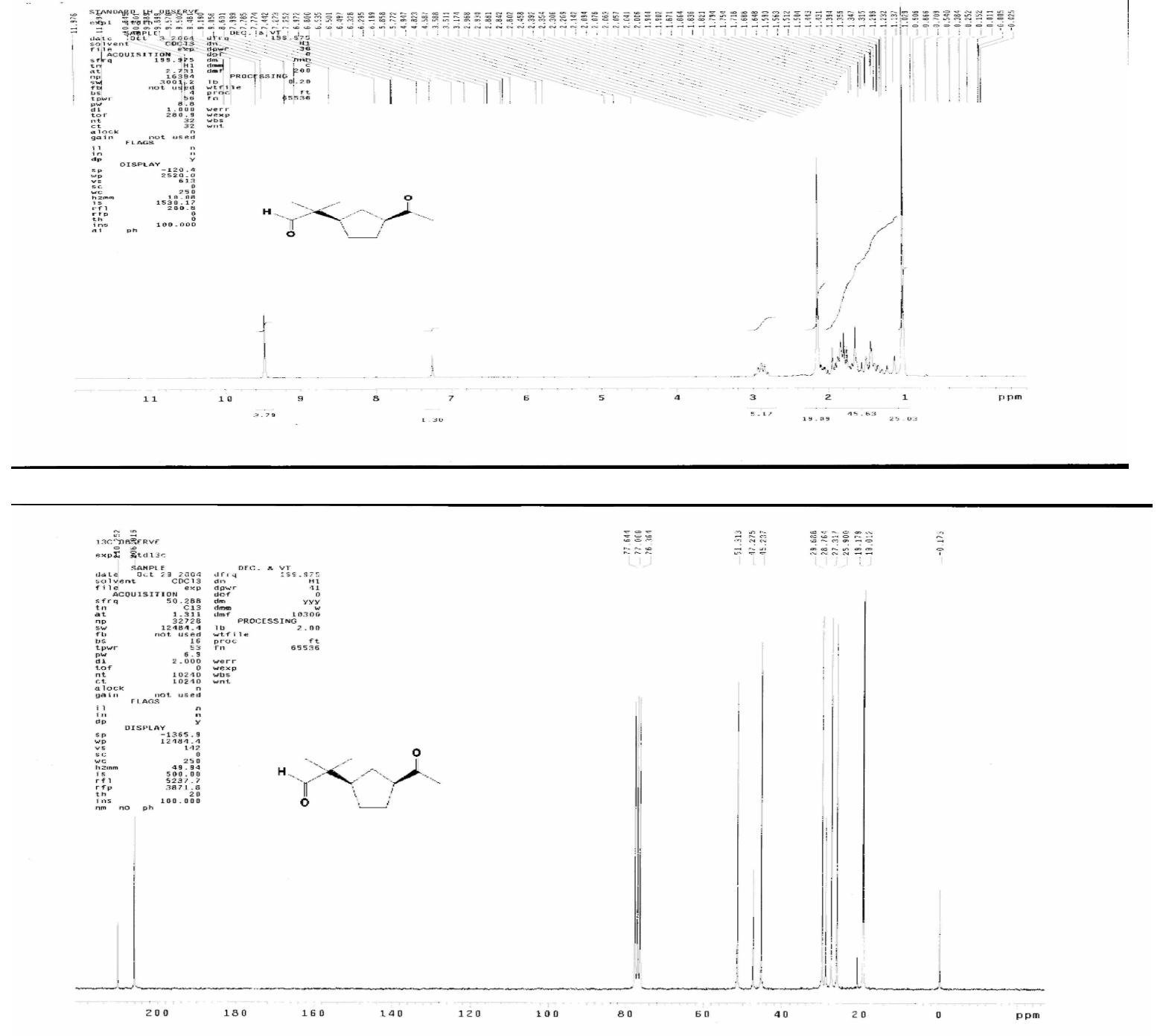
Cyclopentane derivative 25, a syrup: $\mathrm{R}_{\mathrm{f}}=0.60$ (3:1, hexane/EtOAc);<smiles>CCC(=O)[C@@H]1CC[C@H](C(C)(C)C=O)C1</smiles>

$[\alpha]_{\mathrm{D}}{ }^{27}=+6.2\left(0.01, \mathrm{CH}_{2} \mathrm{Cl}_{2}\right)$; IR(film): 2966, 2875, 2700, 1712, 1465, $1366 \mathrm{~cm}^{-1} ;{ }^{1} \mathrm{H}$ NMR (200 $\left.\mathrm{MHz} \mathrm{CDCl}_{3}\right): \delta 9.5(\mathrm{~s}, 1 \mathrm{H}), 2.94(\mathrm{~m}, 1 \mathrm{H}), 2.48(\mathrm{q}, \mathrm{J}=7.4 \mathrm{~Hz}, 2 \mathrm{H}), 2.12(\mathrm{~m}, 1 \mathrm{H}), 1.97-1.35(\mathrm{~m}$, $6 \mathrm{H}), 1.07$ (t, J=7.4 Hz, 3H), 1.07 (s, 6H); ${ }^{13} \mathrm{C}$ NMR (50 MHz, $\left.\mathrm{CDCl}_{3}\right): \delta 213.0,206.1,50.3,47.4$, 45.4, 34.9, 30.1, 27.9, 26.0, 19.3, 19.1, 7.8. HRMS calcd for $\mathrm{C}_{12} \mathrm{H}_{20} \mathrm{O}_{2}$ : 196.1463; found: 196.1466.
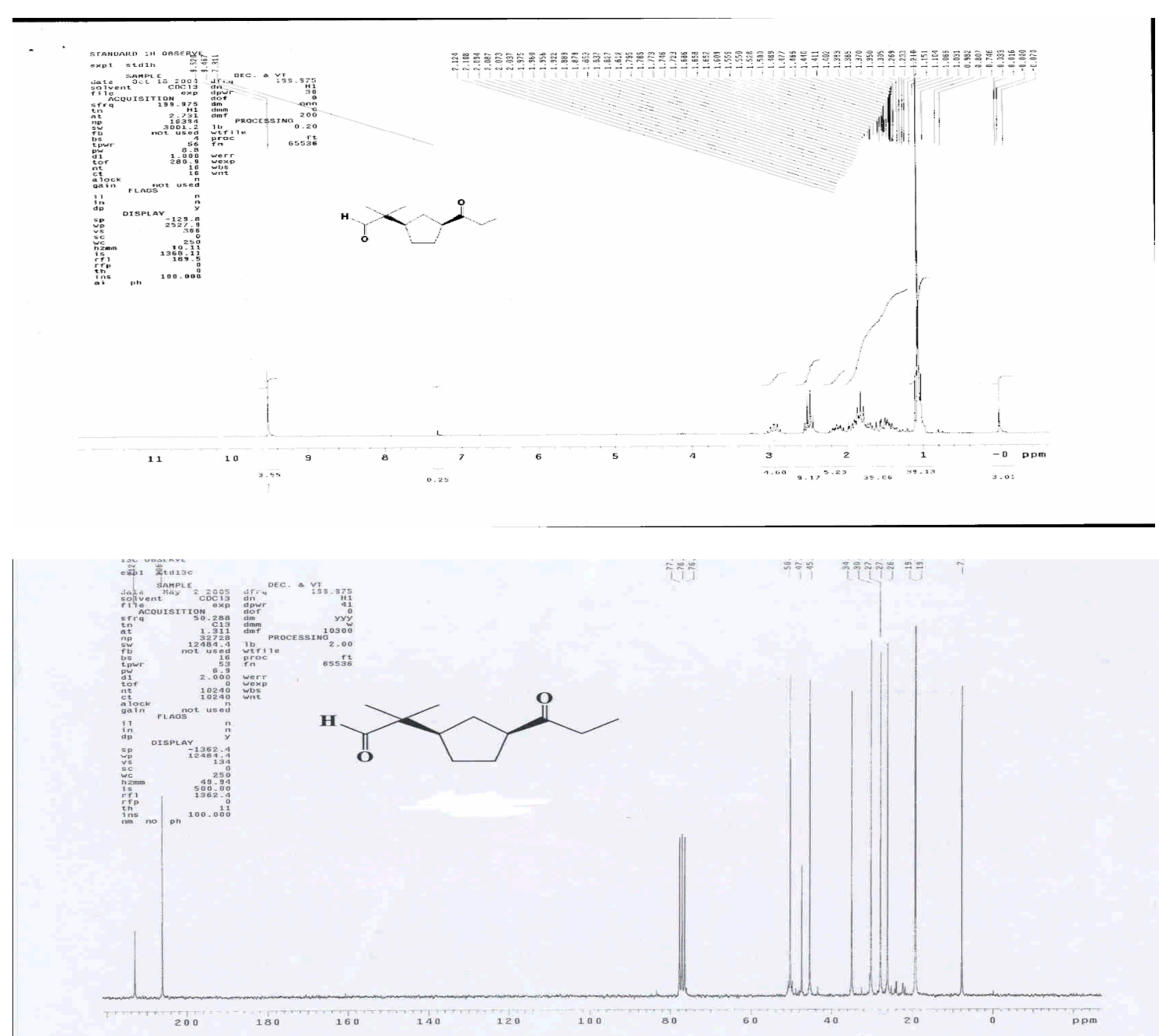
Cyclopentane derivative 26, a syrup: $\mathrm{R}_{\mathrm{f}}=0.49$ (6:1, hexane/EtOAc);

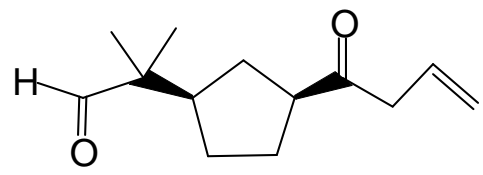

$[\alpha]_{\mathrm{D}}{ }^{27}=+1.8\left(0.01, \mathrm{CH}_{2} \mathrm{Cl}_{2}\right)$; IR(film): 3082, 2964, 2816, 2706, 1715, 1472, $1366 \mathrm{~cm}^{-1} ;{ }^{1} \mathrm{H}$ NMR $\left(200 \mathrm{MHz}, \mathrm{CDCl}_{3}\right): \delta 9.41(\mathrm{~s}, 1 \mathrm{H}), 5.81(\mathrm{~m}, 1 \mathrm{H}), 5.12-4.98(\mathrm{~m}, 2 \mathrm{H}), 3.16-3.11(\mathrm{~m}, 2 \mathrm{H}), 2.89(\mathrm{~m}$, $1 \mathrm{H}), 2.01(\mathrm{~m}, 1 \mathrm{H}), 1.86-1.25(\mathrm{~m}, 6 \mathrm{H}), 0.96(\mathrm{~s}, 6 \mathrm{H}) ;{ }^{13} \mathrm{C} \mathrm{NMR}\left(50 \mathrm{MHz}, \mathrm{CDCl}_{3}\right): \delta 209.9,205.9$, 130.5, 118.4, 50.0, 47.2, 46.5, 45.2, 29.8, 27.5, 25.9, 19.1, 19.0. HRMS calcd for $\mathrm{C}_{13} \mathrm{H}_{20} \mathrm{O}_{2}$ : 208.1463; found: 208.1468 .
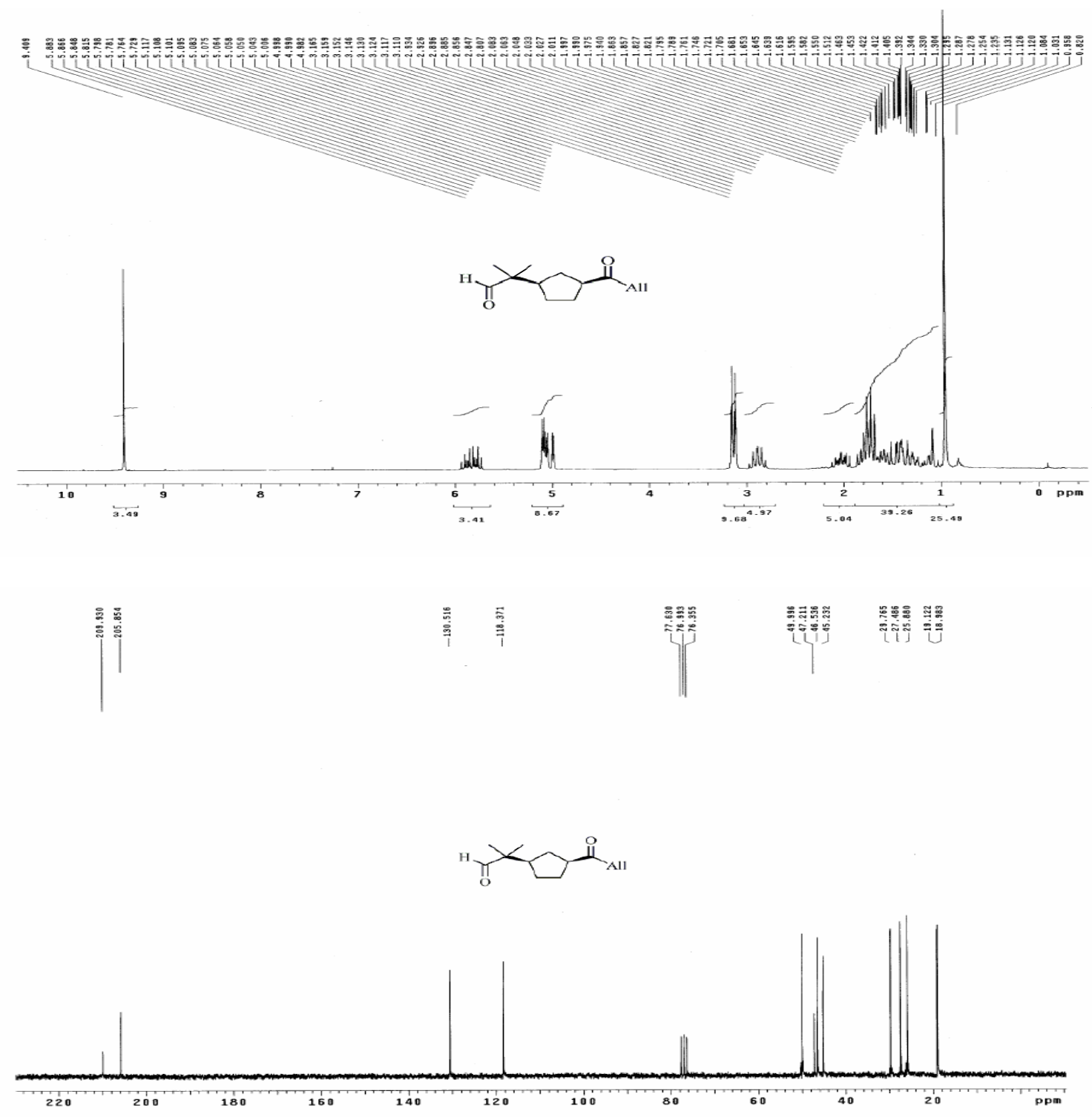
Cyclopentane derivative 27, a syrup: $\mathrm{R}_{\mathrm{f}}=0.49$ (6:1, hexane/EtOAc);<smiles>CCCCC(=O)[C@H]1CC[C@H](C(C)(C)C=O)C1</smiles>

$[\alpha]_{\mathrm{D}}{ }^{27}=+2.0\left(0.01, \mathrm{CH}_{2} \mathrm{Cl}_{2}\right) ; \mathrm{IR}($ film $): 2964,2870,2703,1712,1464,1366 \mathrm{~cm}^{-1} ;{ }^{1} \mathrm{H} \mathrm{NMR}(200$ $\mathrm{MHz}_{\mathrm{CDCl}}$ ): $\delta 9.50(\mathrm{~s}, 1 \mathrm{H}), 2.90(\mathrm{~m}, 1 \mathrm{H}), 2.43(\mathrm{t}, \mathrm{J}=7.2 \mathrm{~Hz}, 2 \mathrm{H}), 2.09(\mathrm{~m}, 1 \mathrm{H}), 1.93-1.21(\mathrm{~m}$, $10 \mathrm{H}), 1.01(\mathrm{~s}, 6 \mathrm{H}), 0.90(\mathrm{t}, \mathrm{J}=7.2 \mathrm{~Hz}, 3 \mathrm{H}) ;{ }^{13} \mathrm{C} \mathrm{NMR}\left(50 \mathrm{MHz}, \mathrm{CDCl}_{3}\right): \delta 212.5,206.0,50.5,47.3$, 45.4, 41.5, 29.9, 27.6, 26.0, 25.7, 22.3, 19.2, 19.1, 13.7. HRMS calcd for $\mathrm{C}_{14} \mathrm{H}_{24} \mathrm{O}_{2}$ : 224.1776; found: 224.1770 .

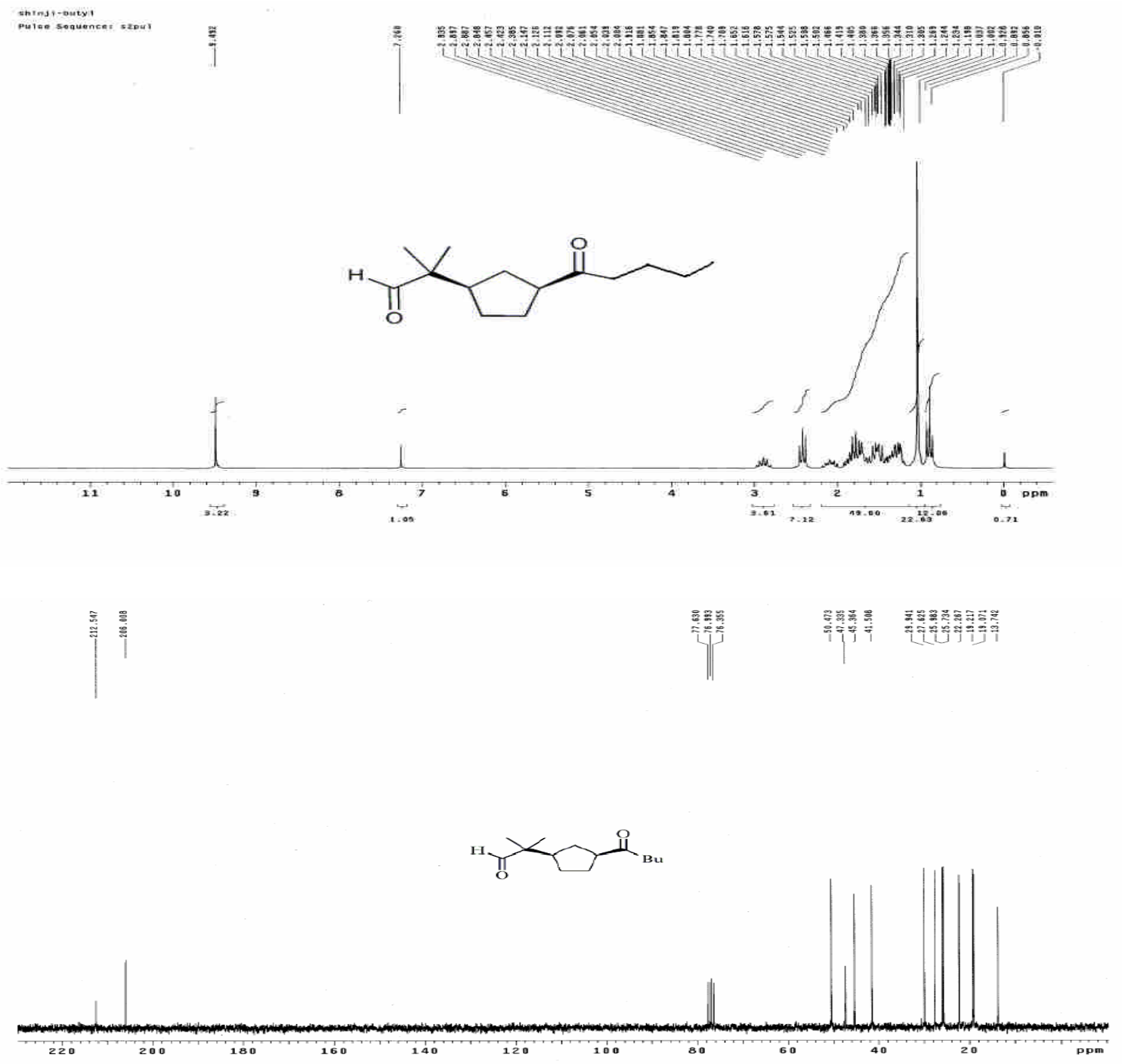


Cyclopentane derivative 28, a syrup: $\mathrm{R}_{\mathrm{f}}=0.55$ (3:1, hexane/EtOAc);<smiles>CC(C)(C=O)[C@H]1CC[C@@H](C(=O)c2ccccc2)C1</smiles>

$[\alpha]_{\mathrm{D}}{ }^{27}=+1.6\left(0.01, \mathrm{CH}_{2} \mathrm{Cl}_{2}\right)$; IR(film): 3055, 2964, 2869, 2704, 1724, 1594, 1449, $1220 \mathrm{~cm}^{-1} ;{ }^{1} \mathrm{H}$ NMR $\left(200 \mathrm{MHz}, \mathrm{CDCl}_{3}\right): \delta 9.53(\mathrm{~s}, 1 \mathrm{H}), 7.98-7.93(\mathrm{~m}, 2 \mathrm{H}), 7.56-7.42(\mathrm{~m}, 3 \mathrm{H}), 3.75(\mathrm{~m}, 1 \mathrm{H})$, $2.12(\mathrm{~m}, 1 \mathrm{H}), 2.04-1.35(\mathrm{~m}, 6 \mathrm{H}), 1.08(\mathrm{~s}, 3 \mathrm{H}), 1.07(\mathrm{~s}, 3 \mathrm{H}) ;{ }^{13} \mathrm{C} \mathrm{NMR}\left(50 \mathrm{MHz}, \mathrm{CDCl}_{3}\right): \delta 206.4$, 202.1, 136.9, 133.1, 128.8, 128.6, 47.7, 46.0, 45.9, 31.0, 29.3, 26.7, 19.6, 19.4. HRMS calcd for $\mathrm{C}_{16} \mathrm{H}_{20} \mathrm{O}_{2}$ : 244.1463; found: 244.1466.

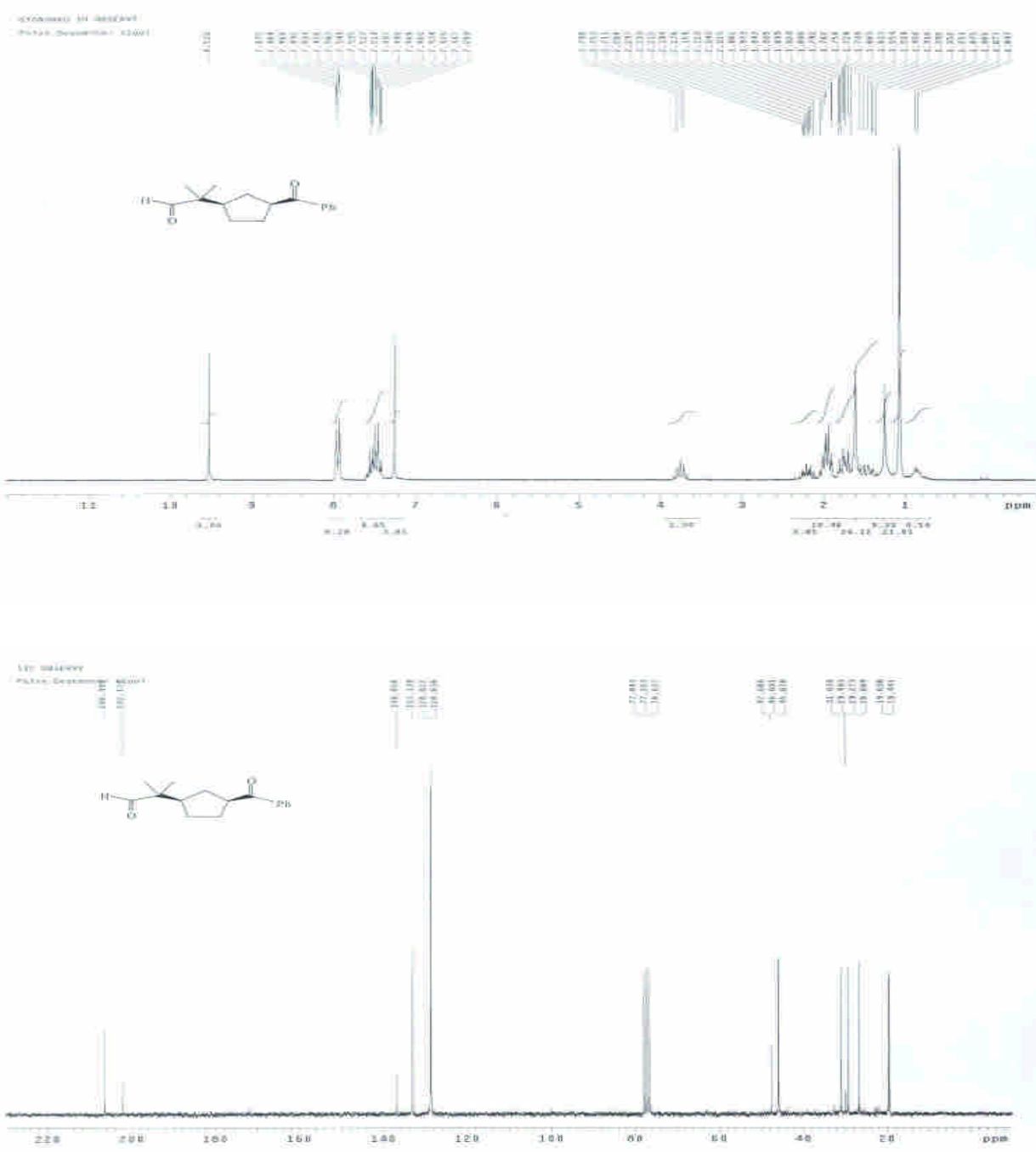




\section{References:}

1. Huckel, W.; Schneider, H. -J.; Schneider-Bernlohr, H. Liebigs Ann. Chem. 1975, 1690-1699.

2. Yang, T. -F.; Zhang, Z. -N.; Tseng, C. -H.; Chen, L. -H. Tetrahedron Lett. 2005, 46, 1917-1920. 